NBI-HE-92-89

PAR-LPTHE-92-49

FTUAM 92-44

November 1992

\title{
Matrix model calculations beyond the spherical limit
}

\author{
J. Ambjørn \\ The Niels Bohr Institute \\ Blegdamsvej 17, DK-2100 Copenhagen Ø, Denmark \\ L. Chekhov日 \\ L.P.T.H.E., Universitè Pierre et Marie Curie \\ 4, pl. Jussieu, 75252, Paris Cedex 05, France \\ C. F. Kristjansen \\ The Niels Bohr Institute \\ Blegdamsvej 17, DK-2100 Copenhagen Ø, Denmark \\ Yu. Makeenko \\ Institute of Theoretical and Experimental Physics \\ B.Cheremushkinskaya 25, 117259 Moscow, Russia
}

\begin{abstract}
We propose an improved iterative scheme for calculating higher genus contributions to the multi-loop (or multi-point) correlators and the partition function of the hermitian one matrix model. We present explicit results up to genus two. We develop a version which gives directly the result in the double scaling limit and present explicit results up to genus four. Using the latter version we prove that the hermitian and the complex matrix model are equivalent in the double scaling limit and that in this limit they are both equivalent to the Kontsevich model. We discuss how our results away from the double scaling limit are related to the structure of moduli space.
\end{abstract}

\footnotetext{
${ }^{1}$ Permanent address: Steklov Mathematical Institute, Vavilov st.42, GSP-1 117966 Moscow, Russia
} 


\section{Introduction}

The hermitian one matrix model is interesting for many reasons. The matrices involved being $N \times N$, the model can be solved in the limit when $N \rightarrow \infty$, as was first demonstrated in Ref. [1]. Furthermore the model admits a $1 / N^{2}$-expansion. This expansion is the simplest example of the 't Hooft topological (or genus) expansion [2] - in the given case of a $D=0$ dimensional quantum field theory. If the leading order (planar or genus zero approximation) result for some quantity, say the vacuum energy, is normalized to be of order 1, the genus $g$ contribution is of order $\mathcal{O}\left(N^{-2 g}\right)$. Even though the hermitian matrix model is often considered a toy model, its perturbative expansion reveals the combinatorial problems one will encounter in the multi-dimensional case: The sum of graphs contributing to a given order of the genus expansion is convergent, while the attempt of summing over all genera leads to a divergence. This was one of the original motivations for studying the genus expansion of the hermitian matrix model.

One more application of the hermitian matrix model is due to the fact that it describes [3] discretized 2-dimensional random surfaces and hence 2D quantum gravity. In this language a diagram of genus $g$ is equivalent to a (piecewise linear) surface of genus $g$. The hermitian one matrix model was extensively studied from this point of view after the observation [4] that it might not only describe pure 2D gravity but also some minimal conformal field theories coupled to 2D quantum gravity $\mathrm{A}$. The relation given in [4] for genus zero was extended to any order of the genus expansion in the socalled double scaling limit, where the coupling constants approach some critical values in a $N$-dependent way [5].

In the double scaling limit many interesting approaches have been developed in order to deal with higher genus contributions. The technique of exactly integrable systems (KdV and KP hierarchies) was applied to the model in Ref. [6]. It provides an algorithm for genus by genus calculations in the double scaling limit. Another approach proposed by Witten [7] is based on the interpretation of the double scaling limit of the matrix model as a topological theory. The problem is then reduced to calculating intersection indices on moduli space. In this approach it was possible to obtain explicit results in the double scaling limit up to genus four [8]. A further development of this approach was the representation [9] of the generating function for the intersection indices as a hermitian one matrix model in an external field (the Kontsevich model).

For the hermitian matrix model away from the double scaling limit, the original genus zero solution [1] was obtained by solving the large- $\mathrm{N}$ integral saddle point equation for the spectral density. To extend the results to higher genera, the orthogonal

\footnotetext{
${ }^{2}$ Although the relation conjectured in 四 turned out to be wrong, the paper was nevertheless instrumental for the later developments.
} 
polynomial technique was applied in Ref. [10]. While this method works well for the partition function in the case of simple potentials and lower genera, it is difficult to implement if one wants an explicit calculation of multi-point correlators and if the potential is "generic".

Another method of solving the hermitian matrix model is based on the loop equations [11] which were first written down for the hermitian matrix model in Ref. [12]. In this approach one solves the genus zero equation and uses the solution in an iterative genus by genus procedure. In the scheme originally proposed in Ref. [11] and elaborated in Ref. [13], one determines the higher genera contributions by solving algebraically the higher loop equations and imposing on all correlators the same analyticity structure as that of their genus zero versions. Following this line of action genus one correlators for $\phi^{4}$ and $\phi^{6}$ potentials were calculated in ref. [14. However, this method involves the entire chain of loop equations and is in practise only applicable when one only wants to calculate a few terms in the genus expansion for a potential with a small number of terms.

An alternative iterative procedure for calculating higher genus contributions to the correlators of the the hermitian matrix model has been proposed recently [15]. It is based only on the first in the chain of loop equations, is applicable for an arbitrary potential and can be pursued order by order of the genus expansion without any problems. A key point in this scheme is a change of variables to the so called moments of the potential. The scheme is close in spirit to the iterative solution of the unitary and hermitian (with cubic potential) one matrix models in an external field [16] $]^{3}$. The genus one partition function and correlators were calculated for an even potential in Ref. [18] and for an arbitrary potential in Ref. [15], where it was also proven that genus $g$ contribution to the $n$-loop correlator depends on at most $2 \times(3 g-2+s)$ lower moments. An analogous algorithm for the complex matrix model was developed in Ref. 19 and the genus 2 and 3 correlators were explicitly calculated for an arbitrary potential.

In the present paper we propose an improved algorithm for an iterative calculation of higher genus contributions to the multi-loop correlators and to the partition function of the hermitian one matrix model. We perform calculations up to and including genus two. Our algorithm differs from that of Ref. [15] by a redefinition of the moments, which has the advantage that it allows us to develop a version which gives directly the result in the double scaling limit where we perform explicit calculations up to and including genus four. We compare the analysis to that of the complex matrix model and prove in particular that the hermitian and complex matrix models are equivalent in

\footnotetext{
${ }^{3}$ For the Kontsevich model similar moments has been used in Ref. 17.
} 
the double scaling limit. We formulate a limiting procedure which allows us to obtain the Kontsevich from the hermitian matrix model and use it to prove in a very direct manner the equivalence between the double scaling limit of the hermitian matrix model and the Kontsevich model.

We discuss a possible interpretation of our results for the hermitian matrix model as representing a particular discretization of moduli space which preserves basic geometrical properties such as intersection indices, and which allows for a detailed study of the boundary of moduli space [20]. We conjecture that the moment-technique developed in the present paper might be an efficient tool in the study of this "fine structure" of moduli space.

The paper is organized as follows: In Section 2 we introduce the basic ingredients of the iterative procedure, the loop insertion operator, the loop equation and the new variables - the moments. In Section 3 we describe the iterative procedure and present the results for the average of loop operators and the partition function for the hermitian matrix model up to genus two. In Section 4 we demonstrate in detail how our iterative procedure works in the double scaling limit and calculate the correlators and the partition function up to genus four. In Section 5 we show that the Kontsevich model can be obtained as a certain limit of the hermitian matrix model, prove the equivalence of the double scaling limit of the hermitian matrix model and the Kontsevich model and discuss the possible connection between our results for the hermitian matrix model away from the double scaling limit and the structure of the discretized

moduli space. Finally Section 6 contains a short discussion of future perspectives and in the Appendix we develop a double scaling version of the iterative procedure for the complex matrix model proposed in [19], and prove that the double scaling limits of the complex and hermitian models coincide.

\section{Main definitions}

The iterative procedure we are going to describe is based on three main ingredients, the loop insertion operator, the first in the chain of loop equations of our model and a suitable change of variables. It allows us to calculate the genus $g$ contribution to the $n$-loop correlator for (in principle) any $g$ and any $n$ and (also in practise) for any potential. The possibility of going to an arbitrarily high genus is provided by the loop equation while the possibility of considering an arbitrarily high number of loops is provided by the loop insertion operator. The change of variables allows us to consider an arbitrary potential. 


\subsection{Loop insertion operator}

The hermitian one matrix model is defined by the partition function

$$
Z[g, N]=e^{N^{2} F}=\int_{N \times N} d \phi \exp (-N \operatorname{Tr} V(\phi))
$$

where the integration is over hermitian $N \times N$ matrices and

$$
V(\phi)=\sum_{j=1}^{\infty} \frac{g_{j}}{j} \phi^{j}
$$

Expectation values (or averages) are defined in the usual way as

$$
\langle Q(\phi)\rangle=\frac{1}{Z} \int d \phi \exp (-N \operatorname{Tr} V(\phi)) Q(\phi) .
$$

We introduce the generating functional (the 1-loop average)

$$
W(p)=\frac{1}{N} \sum_{k=0}^{\infty}\left\langle\operatorname{Tr} \phi^{k}\right\rangle / p^{k+1}
$$

and the $s$-loop correlator $(s \geq 2)$

$$
W\left(p_{1}, \ldots, p_{s}\right)=N^{s-2} \sum_{k_{1}, \ldots, k_{s}=1}^{\infty}\left\langle\operatorname{Tr} \phi^{k_{1}} \ldots \operatorname{Tr} \phi^{k_{s}}\right\rangle_{c o n n .} / p_{1}^{k_{1}+1} \ldots p_{s}^{k_{s}+1}
$$

where conn. refers to the connected part. One can rewrite the last two equations as follows

$$
W\left(p_{1}, \ldots, p_{s}\right)=N^{s-2}\left\langle\operatorname{Tr} \frac{1}{p_{1}-\phi} \ldots \operatorname{Tr} \frac{1}{p_{s}-\phi}\right\rangle_{\text {conn } .} .
$$

As is explained in Ref. [4], these quantities are associated with the (Laplace images of the) sum over discretized open surfaces with $s$ boundaries.

The correlators can be obtained from the free energy, $F$, by application of the loop insertion operator, $\frac{d}{d V(p)}$ :

$$
W\left(p_{1}, \ldots, p_{s}\right)=\frac{d}{d V\left(p_{s}\right)} \frac{d}{d V\left(p_{s-1}\right)} \ldots \frac{d F}{d V\left(p_{1}\right)}
$$

where

$$
\frac{d}{d V(p)} \equiv-\sum_{j=1}^{\infty} \frac{j}{p^{j+1}} \frac{d}{d g_{j}} .
$$

Notice that Eq. (2.7) can be rewritten as

$$
W\left(p_{1}, \ldots, p_{s}\right)=\frac{d}{d V\left(p_{s}\right)} \frac{d}{d V\left(p_{s-1}\right)} \ldots \frac{d}{d V\left(p_{2}\right)} W\left(p_{1}\right)
$$


which shows that if the 1-loop correlator is known for an arbitrary potential, all multiloop correlators can be calculated. This is why it is named above as the generating functional.

With the normalization chosen above, the genus expansion of the correlators reads

$$
W\left(p_{1}, \ldots, p_{s}\right)=\sum_{g=0}^{\infty} \frac{1}{N^{2 g}} W_{g}\left(p_{1}, \ldots, p_{s}\right) \quad(s \geq 1) .
$$

Similarly we have

$$
F=\sum_{g=0}^{\infty} \frac{1}{N^{2 g}} F_{g}
$$

for the genus expansion of the free energy.

\subsection{The loop equation}

The first in the chain of loop equations for the hermitian matrix model can conveniently be written as [21]

$$
\oint_{C} \frac{d \omega}{2 \pi i} \frac{V^{\prime}(\omega)}{p-\omega} W(\omega)=(W(p))^{2}+\frac{1}{N^{2}} W(p, p)
$$

where $V(\omega)=\sum_{j} g_{j} \omega^{j} / j$ and $C$ is a curve which encloses the singularities of $W(\omega)$ and not the point $\omega=p$. This contour integration acts as a projector picking up the coefficients of $p^{-1}$. Due to Eq. (2.9) the second term on the right hand side of the loop equation (2.12) is expressed via $W(p)$, so that (2.12) is a closed equation which determines this quantity.

To leading order in $1 / N^{2}$ one can ignore the last term in Eq. (2.12) and assuming that the singularities of $W(\omega)$ consist of only one cut on the real axis and that $W(p)$ behaves as $1 / p$ as $p \rightarrow \infty$ one finds 11

$$
W_{0}(p)=\frac{1}{2} \oint_{C} \frac{d \omega}{2 \pi i} \frac{V^{\prime}(\omega)}{p-\omega}\left\{\frac{(p-x)(p-y)}{(\omega-x)(\omega-y)}\right\}^{1 / 2}
$$

where $x$ and $y$ are determined by the matrix model potential in the following way

$$
\oint_{C} \frac{d \omega}{2 \pi i} \frac{V^{\prime}(\omega)}{\sqrt{(\omega-x)(\omega-y)}}=0, \quad \oint_{C} \frac{d \omega}{2 \pi i} \frac{\omega V^{\prime}(\omega)}{\sqrt{(\omega-x)(\omega-y)}}=2 .
$$

This genus zero solution will be used below in the iterative procedure of solving the loop equation.

Inserting the genus expansion (2.10) in Eq. (2.12) it appears that $W_{g}(p), g \geq 1$, obeys the following equation

$$
\left\{\hat{K}-2 W_{0}(p)\right\} W_{g}(p)=\sum_{g^{\prime}=1}^{g-1} W_{g^{\prime}}(p) W_{g-g^{\prime}}(p)+\frac{d}{d V(p)} W_{g-1}(p)
$$


where $\hat{K}$ is a linear operator, namely

$$
\hat{K} f(p) \equiv \oint_{C} \frac{d \omega}{2 \pi i} \frac{V^{\prime}(\omega)}{p-\omega} f(\omega) .
$$

In Eq. (2.15) $W_{g}(p)$ is expressed entirely in terms of $W_{g_{i}}(p), g_{i}<g$. This is what makes it possible to develop the iterative procedure mentioned in the Introduction.

\subsection{The new variables}

To characterize the matrix model potential we introduce instead of the couplings $g_{j}$ the moments $M_{k}$ and $J_{k}$ defined by

$$
\begin{aligned}
M_{k} & =\oint_{C} \frac{d \omega}{2 \pi i} \frac{V^{\prime}(\omega)}{(\omega-x)^{k+1 / 2}(\omega-y)^{1 / 2}} & & k \geq 1, \\
J_{k} & =\oint_{C} \frac{d \omega}{2 \pi i} \frac{V^{\prime}(\omega)}{(\omega-x)^{1 / 2}(\omega-y)^{k+1 / 2}} & & k \geq 1 .
\end{aligned}
$$

These moments depend on the coupling constants $g_{j}$ 's both explicitly and via $x$ and $y$ which are determined by Eq. (2.14):

$$
\begin{aligned}
M_{k} & =g_{k}+\left(\frac{1}{2} x+\left(k+\frac{1}{2}\right) y\right) g_{k+1}+\ldots \\
J_{k} & =g_{k}+\left(\left(k+\frac{1}{2}\right) x+\frac{1}{2} y\right) g_{k+1}+\ldots
\end{aligned}
$$

Notice that $M_{k}$ and $J_{k}$ depend explicitly only on $g_{j}$ with $j \geq k$.

There are several motivations for introducing these new variables. First, as we shall see below, for each term in the genus expansion of the partition function and the correlators, the dependence on the infinite set of coupling constants will arrange into a simple function of a finte number of the moments. Moreover, these new variables reflect more directly than the coupling constants the possible critical behaviour of the matrix model. Let us briefly describe how this comes about.

Performing the contour integral in (2.13) by taking residuals at $\omega=p$ and $\omega=\infty$ one finds

$$
W_{0}(p)=\frac{1}{2}\left\{V^{\prime}(p)-M(p) \sqrt{(p-x)(p-y)}\right\}
$$

where $M(p)$ is a polynomial in $p$ of degree two less than $V(p)$. As already discussed in the Introduction, $W_{0}(p)$ can also be determined by an analysis of the matrix model in the eigenvalue picture [1]. Requiring that $W_{0}(p)$ is analytic in the complex plane except for a branch cut $[y, x]$ on the real axis and behaves as $1 / p$ for $p \rightarrow \infty$ corresponds to requiring that the eigenvalue density, $\rho(\lambda)$, has support only on the interval $[y, x]$ and is normalized to 1 . The eigenvalue density is in this situation given by

$$
\rho(\lambda)=\frac{1}{\pi} M(\lambda) \sqrt{(\lambda-y)(x-\lambda)} \quad y \leq \lambda \leq x .
$$


This function vanishes under normal circumstances as a square root at both ends of its support. Critical behaviour arises when some of the roots of $M(\lambda)$ approach $x$ or $y$. For a non-symmetric potential the so called $m^{t h}$ multi-critical point is reached when $(m-1)$ extra zeros accumulate at either $x$ or $y$. Comparing (2.13) and (2.21) it appears that

$$
\left.M_{k} \propto \frac{d^{(k-1)} M(\lambda)}{d \lambda^{k-1}}\right|_{\lambda=x} \quad,\left.\quad J_{k} \propto \frac{d^{(k-1)} M(\lambda)}{d \lambda^{k-1}}\right|_{\lambda=y}
$$

so the condition for being at the $m^{\text {th }}$ multi-critical point is simply $M_{1}=M_{2}=\ldots=$ $M_{k-1}=0$ and $M_{k} \neq 0$ if the extra zeros accumulate at $x$ and $J_{1}=J_{2}=\ldots=J_{k-1}=0$, $J_{k} \neq 0$ if the extra zeros accumulate at $y$. For the symmetric potential $(V(\phi)=V(-\phi))$ the eigenvalues will be distributed symmetrically around zero and hence confined to an interval of the type $[-\sqrt{z}, \sqrt{z}]$ in the one arc case. In a situation like this the $m^{\text {th }}$ multi-critical point is characterized by the eigenvalue density having $(m-1)$ extra zeros at both $-\sqrt{z}$ and $\sqrt{z}$. Reexamining (2.17) and (2.18) it appears that for the symmetric potential

$$
M_{k}=(-1)^{k+1} J_{k}, \quad(V(\phi)=V(-\phi)) .
$$

Hence in this case we have only one set of moments and the condition for being at the $m^{\text {th }}$ multi-critical point is the vanishing of the first $(m-1)$ of these.

This formalism obviously allows for a treatment of a more general situation where $m$ extra zeros accumulate at $x$ and $n$ extra zeros accumulate at $y$. Such multi-critical models have been studied in Ref. [22]. We will however restrict ourselves to the traditional models.

The superiority of the moments defined in (2.17) and (2.18) as compared to the coupling constants will become even more clear when we consider the double scaling limit in Section 4.1. By then it will also become evident why these new moments are more convenient than those originally introduced in Ref. [15].

\section{The iterative procedure}

Our iterative solution of the loop equation results in a certain representation of the free energy and the correlators in terms of the moments. In this section we describe the structure of $W_{g}(p)$ and $F_{g}$ and show that the iterative procedure can be conveniently

formulated by referring to the eigenvectors of a linear operator, $\hat{K}$. We prove that the genus $g$ contribution to the $s$-loop correlator depends at most on $2 \times(3 g-2+s)$ lower moments $(2 \times(3 g-2)$ for the partition function) for $g>0$. We perform explicit calculations up to genus two. 


\subsection{The structure of $F_{g}$ and $W_{g}(p)$}

Our iterative procedure of solving the loop equation results in the following representation of the genus $g$ contribution to the free energy

$$
F_{g}=\sum_{\substack{\alpha_{j}>1, \beta_{i}>1}}\left\langle\alpha_{1} \ldots \alpha_{s} ; \beta_{1} \ldots \beta_{l} \mid \alpha, \beta, \gamma\right\rangle_{g} \frac{M_{\alpha_{1}} \ldots M_{\alpha_{s}} J_{\beta_{1}} \ldots J_{\beta_{l}}}{M_{1}^{\alpha} J_{1}^{\beta} d^{\gamma}} \quad g \geq 1
$$

where $d=x-y$ is the distance between the endpoints of the support of the eigenvalue distribution, the brackets denote rational numbers and $\alpha, \beta$ and $\gamma$ are non-negative integers. The indices $\alpha_{1}, \ldots, \alpha_{s}, \beta_{1}, \ldots, \beta_{l}$ take values in the interval $[2,3 g-2]$ and the summation is over sets of indices. In particular $F_{g}$ depends on at most $2 \times(3 g-2)$ moments. Furthermore, since nothing allows us to distinguish between $x$ and $y, F_{g}$ must be invariant under the interchange of the two. Hence one gets

$$
F: \quad\left\langle\alpha_{1} \ldots \alpha_{s} ; \beta_{1} \ldots \beta_{l} \mid \alpha, \beta, \gamma\right\rangle=(-1)^{\gamma}\left\langle\beta_{1} \ldots \beta_{l} ; \alpha_{1} \ldots \alpha_{s} \mid \beta, \alpha, \gamma\right\rangle
$$

There are certain restrictions on the integers which enter Eq. (3.1). Let us denote by $N_{M}$ and $N_{J}$ the total powers of $M$ 's and $J$ 's, respectively, i.e.

$$
N_{M}=s-\alpha, \quad N_{J}=l-\beta .
$$

Then it holds that

$$
N_{M} \leq 0, \quad N_{J} \leq 0
$$

and

$$
\begin{array}{cc}
F_{g}: & N_{M}+N_{J}=2-2 g \\
F_{g}: & \sum_{i=1}^{s}\left(\alpha_{i}-1\right)+\sum_{j=1}^{l}\left(\beta_{j}-1\right)+\gamma=4 g-4 .
\end{array}
$$

The relation (3.5) follows from the fact that the partition function $Z=e^{\sum_{g} N^{2-2 g} F_{g}}$ is invariant under simultaneous rescalings of $N$ and the eigenvalue density, $\rho(\lambda) ; N \rightarrow k \cdot N$, $\rho(\lambda) \rightarrow \frac{1}{k} \rho(\lambda)$. The relation (3.6) follows from the invariance of $Z$ under rescalings of the type $N \rightarrow k^{2} \cdot N, g_{j} \rightarrow k^{j-2} g_{j}$. Finally the following inequality must be fulfilled:

$$
F_{g}: \quad \sum_{i=1}^{s}\left(\alpha_{i}-1\right)+\sum_{j=1}^{l}\left(\beta_{j}-1\right) \leq 3 g-3
$$

This requirement becomes more transparent when we consider the double scaling limit in Section 4.1. In combination with Eq. (3.6) it gives

$$
g-1 \leq \gamma \leq 4 g-4
$$


To explain the structure of $W_{g}(p)$, let us introduce the basis vectors $\chi^{(n)}(p)$ and $\Psi^{(n)}(p)$ :

$$
\begin{array}{ll}
\chi^{(n)}(p)=\frac{1}{M_{1}}\left\{\Phi_{x}^{(n)}(p)-\sum_{k=1}^{n-1} \chi^{(k)}(p) M_{n-k+1}\right\} & n \geq 1 \\
\Psi^{(n)}(p)=\frac{1}{J_{1}}\left\{\Phi_{y}^{(n)}(p)-\sum_{k=1}^{n-1} \Psi^{(k)}(p) J_{n-k+1}\right\} & n \geq 1
\end{array}
$$

where

$$
\begin{array}{ll}
\Phi_{x}^{(n)}(p)=(p-x)^{-n}\{(p-x)(p-y)\}^{-1 / 2} & n \geq 0, \\
\Phi_{y}^{(n)}(p)=(p-y)^{-n}\{(p-x)(p-y)\}^{-1 / 2} & n \geq 0 .
\end{array}
$$

It is easy to show for the operator $\hat{K}$ defined by Eq. (2.16) that

$$
\begin{array}{ll}
\left\{\hat{K}-2 W_{0}(p)\right\} \chi^{(n)}(p)=\frac{1}{(p-x)^{n}} & n \geq 1, \\
\left\{\hat{K}-2 W_{0}(p)\right\} \Psi^{(n)}(p)=\frac{1}{(p-y)^{n}} & n \geq 1
\end{array}
$$

and that the kernel of $\left\{\hat{K}-2 W_{0}(p)\right\}$ is spanned by $\Phi_{x}^{(0)}(p)=\Phi_{y}^{(0)}(p)$.

Since $W_{g}(p)$ can be obtained from $F_{g}$ according to Eq. (2.7), the representation (3.1) impliest

$$
W_{g}(p)=\sum_{n=1}^{3 g-1}\left\{A_{g}^{(n)} \chi^{(n)}(p)+D_{g}^{(n)} \Psi^{(n)}(p)\right\} \quad g \geq 1 .
$$

We do not add any multiple of $\Phi_{x}^{(0)}(p)$ or $\Phi_{y}^{(0)}(p)$. Doing so would contradict the boundary condition $W(p) \rightarrow 1 / p$ for $p \rightarrow \infty$ since this behaviour was already obtained for genus zero. We note that this structure of $W_{g}(p)$ is in agreement with the assumption [13] that $W_{g}(p)$ is analytic in the complex plane except for a branch cut $[y, x]$ on the real axis.

The coefficients $A_{g}^{(n)}$ are of the same structure as $F_{g}$ and the relation (3.4) still holds. However in this case the indices $\alpha_{1}, \ldots, \alpha_{s}, \beta_{1}, \ldots, \beta_{l}$ take values in the interval $[2,3 g-n]$. Hence $W_{g}(p)$ depends on at most $2 \times(3 g-1)$ moments. The invariance of the partition function under the rescalings described above has the following implications for the structure of $A_{g}^{(n)}$ :

$$
\begin{array}{cc}
A_{g}^{(n)}: & N_{M}+N_{J}=2-2 g, \\
A_{g}^{(n)}: & \sum_{i=1}^{s}\left(\alpha_{i}-1\right)+\sum_{j=1}^{l}\left(\beta_{j}-1\right)+\gamma=4 g-2-n .
\end{array}
$$

\footnotetext{
${ }^{4} \mathrm{~A}$ similar formula is proven in Ref. 15] for a different definition of the moments and of the basis vectors.
} 
We also have an analogue of (3.7) for $A_{g}^{(n)}$. It reads

$$
A_{g}^{(n)}: \quad \sum_{i=1}^{k}\left(\alpha_{i}-1\right)+\sum_{j=1}^{l}\left(\beta_{j}-1\right) \leq 3 g-n-1 .
$$

Again we refer to Section 4.1 for further explanations. However, we note that combining (3.17) and (3.18) one gets again the bound (3.8) on $\gamma$.

As was the case for $F_{g}, W_{g}(p)$ must be invariant under the interchange of $x$ and $y$. This means that $D_{g}^{(n)}$ must appear from $A_{g}^{(n)}$ by the replacements $d \rightarrow-d, J \leftrightarrow M$. (We note that we do not have a relation like (3.2) for the $A_{g}^{(n)}$ 's.) Furthermore, $W_{g}(p)$ must be an odd function of $p$ for a symmetric potential. This implies that $A_{g}^{(n)}=$ $(-1)^{n} D_{g}^{(n)}$.

That the structure of $F_{g}$ and $W_{g}(p)$ actually is as described in this section can be proven by induction. We will not go through the proof here. Instead we refer to Refs. [15, 19]. In the latter reference a formula somewhat similar to (3.15) was proven for the complex matrix model. However the strategy of the proof will be evident from Sections 3.2 and 3.3 where we describe the iterative procedures which allow us to calculate $W_{g}(p)$ and $F_{g}$ for any $g$ starting from $W_{0}(p)$.

\subsection{The iterative procedure for determining $W_{g}(p)$}

According to Eq. (2.12), we need to calculate $W_{0}(p, p)$ in order to start the iterative procedure. To do this we write the loop insertion operator as

$$
\frac{d}{d V(p)}=\frac{\partial}{\partial V(p)}+\frac{d x}{d V(p)} \frac{\partial}{\partial x}+\frac{d y}{d V(p)} \frac{\partial}{\partial y}
$$

where

$$
\frac{\partial}{\partial V(p)}=-\sum_{j=1}^{\infty} \frac{j}{p^{j+1}} \frac{\partial}{\partial g_{j}} .
$$

The derivatives $d x / d V(p)$ and $d y / d V(p)$ can be obtained from (2.14) and read

$$
\frac{d x}{d V(p)}=\frac{1}{M_{1}} \Phi_{x}^{(1)}, \quad \frac{d y}{d V(p)}=\frac{1}{J_{1}} \Phi_{y}^{(1)} .
$$

Using the relation

$$
\frac{\partial}{\partial V(p)} V^{\prime}(\omega)=\frac{\partial}{\partial p} \frac{1}{p-\omega}
$$

one finds 23]

$$
W_{0}(p, p)=\frac{(x-y)^{2}}{16(p-x)^{2}(p-y)^{2}} .
$$


This enables us to find $W_{1}(p)$ and we see that it is of the form (3.15) with

$$
\begin{array}{cl}
A_{1}^{(1)}=-\frac{1}{8 d}, & A_{1}^{(2)}=\frac{1}{16}, \\
D_{1}^{(1)}=\frac{1}{8 d}, & D_{1}^{(2)}=\frac{1}{16} .
\end{array}
$$

Carrying on the iteration process is straightforward. In each step one must calculate the right hand side of the loop equation (2.12). Decomposing the result obtained into fractions of the type $(p-x)^{-n},(p-y)^{-n}$ allows one to identify immediately the coefficients $A_{g}^{(n)}$ and $D_{g}^{(n)}$.

To calculate $W_{g}(p, p)$ it is convenient to write the loop insertion operator as

$$
\frac{d}{d V(p)}=\sum_{n} \frac{d M_{n}}{d V(p)} \frac{\partial}{\partial M_{n}}+\sum_{j} \frac{d J_{j}}{d V(p)} \frac{\partial}{\partial J_{j}}+\frac{d x}{d V(p)} \frac{\partial}{\partial x}+\frac{d y}{d V(p)} \frac{\partial}{\partial y}
$$

where

$$
\begin{aligned}
\frac{d M_{n}}{d V(p)}= & -\frac{1}{2}(p-x)^{-n-1 / 2}(p-y)^{-3 / 2}-(n+1 / 2) \Phi_{x}^{(n+1)}(p) \\
& +\frac{1}{2}\left\{\sum_{i=1}^{n}(-1)^{n-i} M_{i}\left(\frac{1}{x-y}\right)^{n-i+1}+(-1)^{n} J_{1}\left(\frac{1}{x-y}\right)^{n}\right\} \frac{d y}{d V(p)} \\
& +(n+1 / 2) M_{n+1} \frac{d x}{d V(p)} .
\end{aligned}
$$

The derivatives $d x / d V(p)$ and $d y / d V(p)$ are given by 3.21) and the function $\Phi_{x}^{(n)}$ was defined in (3.11). Of course $d J_{n} / d V(p)$ just appears from $d M_{n} / d V(p)$ by the replacements $y \leftrightarrow x$ and $J \leftrightarrow M$. We note that there is no simplification of the algorithm in the case of the symmetric potential. We can only put $x=-y=\sqrt{z}$ at the end of the calculation. This complication stems of course from the fact that we have to keep the odd coupling constants in the loop insertion operator until all differentiations have been performed. Only hereafter they can be put equal to zero. The same is not true in the double scaling limit however. We will come back to this later.

By taking a closer look at the loop insertion operator (3.26) and bearing in mind the results (3.23), (3.24) and (3.25), it is easy to convince oneself that $A_{g}^{(n)}$ and $D_{g}^{(n)}$ depend only on $x$ and $y$ via $(x-y)$ and have the structure shown in equation (3.1). Furthermore it appears that $W_{g}\left(p_{1}, \ldots, p_{s}\right)$ depends on at most $2 \times(3 g-2+s)$ parameters. The 
results for $g=2$ obtained with the aid of Mathematica read

$$
\begin{aligned}
& A_{2}^{(1)}=\frac{201}{256 d^{5} J_{1}{ }^{2}}-\frac{67 J_{2}}{128 d^{4} J_{1}{ }^{3}}-\frac{5 J_{3}}{32 d^{3} J_{1}{ }^{3}}+\frac{49 J_{2}{ }^{2}}{256 d^{3} J_{1}{ }^{4}} \\
& +\frac{57}{64 d^{5} J_{1} M_{1}}-\frac{11 J_{2}}{128 d^{4} J_{1}^{2} M_{1}}+\frac{49 M_{2}^{2}}{256 d^{3} M_{1}^{4}} \\
& +\frac{201}{256 d^{5} M_{1}^{2}}+\frac{22 M_{2}}{128 d^{4} J_{1} M_{1}^{2}}-\frac{J_{2} M_{2}}{64 d^{3} J_{1}^{2} M_{1}^{2}} \\
& +\frac{67 M_{2}}{128 d^{4} M_{1}^{3}}-\frac{5 M_{3}}{32 d^{3} M_{1}^{3}} \text {, } \\
& A_{2}^{(2)}=-\frac{57}{128 d^{4} J_{1} M_{1}}+\frac{8 J_{2}}{128 d^{3} J_{1}{ }^{2} M_{1}}-\frac{49 M_{2}{ }^{2}}{256 d^{2} M_{1}{ }^{4}} \\
& -\frac{201}{256 d^{4} M_{1}^{2}}-\frac{3 M_{2}}{128 d^{3} J_{1} M_{1}^{2}}+\frac{J_{2} M_{2}}{128 d^{2}{J_{1}}^{2} M_{1}^{2}} \\
& -\frac{67 M_{2}}{128 d^{3} M_{1}^{3}}+\frac{5 M_{3}}{32 d^{3} M_{1}^{3}} \text {, } \\
& A_{2}^{(3)}=\frac{49 M_{2}{ }^{2}}{256 d M_{1}{ }^{4}}-\frac{5 M_{3}}{32 d M_{1}{ }^{3}}+\frac{67 M_{2}}{128 d^{2} M_{1}{ }^{3}} \\
& +\frac{201}{256 d^{3} M_{1}^{2}}+\frac{15}{128 d^{3} J_{1} M_{1}}-\frac{5 J_{2}}{128 d^{2} J_{1}^{2} M_{1}} \text {, } \\
& A_{2}^{(4)}=-\frac{49 M_{2}}{128 d M_{1}^{3}}-\frac{189}{256 d^{2} M_{1}^{2}} \text {, } \\
& A_{2}^{(5)}=\frac{105}{256 d M_{1}^{2}} \text {; } \\
& D_{2}^{(1)}=A_{2}^{(1)}(M \longleftrightarrow J, d \longrightarrow-d), \\
& D_{2}^{(2)}=A_{2}^{(2)}(M \longleftrightarrow J, d \longrightarrow-d) \text {, } \\
& D_{2}^{(3)}=A_{2}^{(3)}(M \longleftrightarrow J, d \longrightarrow-d) \text {, } \\
& D_{2}^{(4)}=A_{2}^{(4)}(M \longleftrightarrow J, d \longrightarrow-d) \text {, } \\
& D_{2}^{(5)}=A_{2}^{(5)}(M \longleftrightarrow J, d \longrightarrow-d) \text {. }
\end{aligned}
$$

The genus two contribution to $W(p)$ is now determined by Eq. (3.15).

\subsection{The iterative procedure for $F_{g}$}

In this section we present an algorithm which allows one to determine $F_{g}$, as soon as the result for $W_{g}(p)$ is known. The strategy consists in writing the basis vectors $\chi^{(n)}(p)$ and $\Psi^{(n)}(p)$ as derivatives with respect to $V(p)$. It is easy to verify that the following relations hold

$$
\chi^{(1)}(p)=\frac{d x}{d V(p)},
$$




$$
\begin{aligned}
\Psi^{(1)}(p) & =\frac{d y}{d V(p)}, \\
\chi^{(2)}(p) & =\frac{d}{d V(p)}\left\{-\frac{2}{3} \ln M_{1}-\frac{1}{3} \ln d\right\}, \\
\Psi^{(2)}(p) & =\frac{d}{d V(p)}\left\{-\frac{2}{3} \ln J_{1}-\frac{1}{3} \ln d\right\} .
\end{aligned}
$$

Combining this with the results (3.24) and (3.25) one immediately finds

$$
F_{1}=-\frac{1}{24} \ln M_{1}-\frac{1}{24} \ln J_{1}-\frac{1}{6} \ln d
$$

which coincides with the expression of Ref. [15].

In the general case things are not quite as simple. The basis vectors can not be written as total derivatives. This is of course in accordance with the fact that the $A$ and $D$ coefficients now have a more complicated dependence on the potential (cf. Section 3.2). However, a rewriting of the basis vectors allows one to identify relatively simply $W_{g}(p)$ as a total derivative. In the case of $\chi^{(n)}(p)$ this rewriting reads

$$
\begin{aligned}
\chi^{(n)}(p)= & \frac{1}{M_{1}}\left\{-\frac{1}{2 n-1} \sum_{i=1}^{n-1}(-1)^{n-i-1}\left\{\Phi_{x}^{(i)}-M_{i} \frac{d y}{d V(p)}\right\}\left(\frac{1}{x-y}\right)^{n-i}\right. \\
& \left.-\frac{2}{2 n-1} \frac{d M_{n-1}}{d V(p)}-\sum_{k=2}^{n-1} \chi^{(k)} M_{n-k+1}\right\}, \quad n \geq 2
\end{aligned}
$$

where $\Phi_{x}^{(n)}$ should be written as

$$
\begin{aligned}
\Phi_{x}^{(n)}= & -\frac{1}{2 n-1} \sum_{i=1}^{n-1}(-1)^{n-i-1}\left\{\Phi_{x}^{(i)}-M_{i} \frac{d y}{d V(p)}\right\}\left(\frac{1}{x-y}\right)^{n-i} \\
& +M_{n} \frac{d x}{d V(p)}-\frac{2}{2 n-1} \frac{d M_{n-1}}{d V(p)} \quad n \geq 2, \\
\Phi_{x}^{(1)}= & M_{1} \frac{d x}{d V(p)} .
\end{aligned}
$$

The basis vector $\chi^{(1)}(p)$ should of course still be written as in (3.29). The rewriting of the $\Psi^{(n)}$ 's is analogous to that of the $\chi^{(n)}$ 's. It can be obtained by performing the replacements $J \leftrightarrow M$ and $x \leftrightarrow y$ in the formulas above. 
By means of these rewritings we have been able to determine $F_{2}$. The result reads

$$
\begin{aligned}
F_{2}= & -\frac{119}{7680 J_{1}^{2} d^{4}}-\frac{119}{7680 M_{1}^{2} d^{4}}+\frac{181 J_{2}}{480 J_{1}^{3} d^{3}}-\frac{181 M_{2}}{480 M_{1}^{3} d^{3}} \\
& +\frac{3 J_{2}}{64 J_{1}^{2} M_{1} d^{3}}-\frac{3 M_{2}}{64 J_{1} M_{1}^{2} d^{3}}-\frac{11 J_{2}^{2}}{40 J_{1}^{4} d^{2}}-\frac{11 M_{2}^{2}}{40 M_{1}^{4} d^{2}} \\
& +\frac{43 M_{3}}{192 M_{1}^{3} d^{2}}+\frac{43 J_{3}}{192 J_{1}^{3} d^{2}}+\frac{J_{2} M_{2}}{64 J_{1}^{2} M_{1}^{2} d^{2}}-\frac{17}{128 J_{1} M_{1} d^{4}} \\
& +\frac{21 J_{2}^{3}}{160 J_{1}{ }^{5} d}-\frac{29 J_{2} J_{3}}{128 J_{1}^{4} d}+\frac{35 J_{4}}{384 J_{1}^{3} d}-\frac{21 M_{2}^{3}}{160 M_{1}^{5} d} \\
& +\frac{29 M_{2} M_{3}}{128 M_{1}^{4} d}-\frac{35 M_{4}}{384 M_{1}^{3} d} .
\end{aligned}
$$

It is obvious from the formulas above that $F_{g}$ depends for a non-symmetric potential on at most $2 \times(3 g-2)$ moments. Furthermore, for a symmetric potential, $F_{g}$ is a sum of two identical terms and depends on only at most $3 g-2$ moments. A consequence of this doubling for the double scaling limit will be discussed in Section 4.3.

\section{The double scaling limit}

It is easy to determine which terms in the explicit expressions for $F_{g}$ and $W_{g}(p)$ determined in the previous section that contribute in the double scaling limit. However it is rather time consuming to determine $F_{g}$ and $W_{g}(p)$ away from the double scaling limit. In this section we develop an algorithm which gives us directly the result in the double scaling limit. Using this algorithm we calculate the correlators and the partition function explicitly up to genus four and describe their general structure.

\subsection{Multi-critical points}

Let us consider first the case of the symmetric potential. We hence have $x=-y=\sqrt{z}$ and $J_{k}=(-1)^{k+1} M_{k}$ for all values of $k$. As is mentioned earlier, the $m^{\text {th }}$ multi-critical point is characterized by the eigenvalue density having $(m-1)$ extra zeros accumulating at both $-\sqrt{z}$ and $\sqrt{z}$, and the condition for being at this point is the vanishing of the first $(m-1)$ moments. For the $m^{\text {th }}$ multi-critical model the double scaling limit of the correlators is obtained by fixing the ratio of any given coupling and, say $g_{2}$, to its critical value and setting

$$
\begin{aligned}
p^{2} & =z_{c}+a \pi \\
z & =z_{c}-a \Lambda^{1 / m}
\end{aligned}
$$

The moments then scale as

$$
J_{k} \sim M_{k} \sim a^{m-k}, \quad k \in[1, m-1] .
$$


Furthermore, it is well known that the genus $g(g \geq 1)$ contribution to the free energy has the following scaling behaviour

$$
F_{g} \sim a^{(2-2 g)(m+1 / 2)} .
$$

Bearing in mind that the structure of $F_{g}$ is as shown in Eq. (3.1), one finds that the following relation must hold

$$
\sum_{j=1}^{s}\left(m-\alpha_{j}\right)+\sum_{i=1}^{l}\left(m-\beta_{i}\right)-(\alpha+\beta)(m-1) \geq m(2-2 g)-g-1 .
$$

Since the free energy should look the same for all multi-critical models, we have

$$
\begin{gathered}
N_{J}+N_{M} \geq 2-2 g \\
\sum_{j=1}^{s}\left(\alpha_{j}-1\right)+\sum_{i=1}^{l}\left(\beta_{i}-1\right) \leq 3 g-3 .
\end{gathered}
$$

We already know from the analysis in Section 3.1 that the equality sign must hold in (4.6). Only terms for which $\max (s, l) \leq m$ and for which the equality sign holds also in (4.7) will contribute in the double scaling limit. From Eq. (3.6) it follows that these terms will have

$$
\gamma=g-1
$$

A similar analysis can be carried out for the generating functional. Here it is known that the genus $g$ contribution to $W(p)$ has the following scaling behaviour

$$
W_{g}(\pi, \Lambda) \sim a^{(1-2 g)(m+1 / 2)-1}
$$

with the exception of $W_{0}(\pi, \Lambda)$ which also contains a non-scaling part. From (3.9) and $(\overline{3.10})$ it appears that the basis vectors scale as

$$
\chi^{(n)} \sim \Psi^{(n)} \sim a^{-m-n+1 / 2} .
$$

Remembering that the structure of the $A$ and $D$ coefficients is as shown in (3.1), one finds that the following relation must hold for both $A_{g}^{(n)}$ and $D_{g}^{(n)}$

$$
\sum_{j=1}^{s}\left(m-\alpha_{j}\right)+\sum_{i=1}^{l}\left(m-\beta_{i}\right)-(\alpha+\beta)(m-1) \geq m(2-2 g)+n-g-1 .
$$

again provided $\max (s, l) \leq m$. Since also the generating functional should look the same for all multi-critical models, it follows that $A_{g}^{(n)}$ and $D_{g}^{(n)}$ must satisfy the following conditions

$$
N_{J}+N_{M} \geq 2-2 g
$$




$$
\sum_{j=1}^{s}\left(\alpha_{j}-1\right)+\sum_{i=1}^{l}\left(\beta_{i}-1\right) \leq 3 g-n+1 .
$$

Here we recognize the homogeneity requirement (3.18). We know from Section 3.1 that the equality sign always holds in (4.12). Only terms for which $\max (s, l) \leq m$ and for which the equality sign holds also in (4.13) will contribute in the double scaling limit. We note this means that $\gamma=g-1$ for these terms (cf. Eq. (3.17)).

Let us turn now to the case of the non-symmetric potential, and let us consider the $m^{\text {th }}$ multi-critical point assuming that the extra zeros accumulate at $x$. To obtain the double scaling limit of the correlators, we first fix as before the ratio between any given coupling and the first one to its critical value. Then we scale $p$ and $x$ in the following way keeping however $y$ fixed:

$$
\begin{aligned}
& p=x_{c}+a \pi \\
& x=x_{c}-a \Lambda^{1 / m} .
\end{aligned}
$$

Under these circumstances the $J$-moments do not scale but $M_{k}$ behaves as

$$
M_{k} \sim a^{m-k} \quad k \in[1, m-1]
$$

Using again (4.4) we find that the only terms which survive the double scaling limit for $F_{g}$ are those for which

$$
\begin{gathered}
N_{M}=2-2 g, \\
\sum_{j=1}^{s}\left(\alpha_{j}-1\right)=3 g-3 .
\end{gathered}
$$

Comparing Eq. (4.17) with Eq. (3.5), we see that all the $J$-dependence disappears when the prescription for taking the double scaling limit is as in (4.14) and (4.15). Furthermore, comparing (4.18) with (3.6) we find that all remaining terms have $\gamma=$ $g-1$ as in the symmetric case. The relations (4.17) and (4.18) will be exploited in Section 5.2.

Let us carry out the analysis of $W_{g}(p)$ also for the non-symmetric case since this will give us an additional information about the structure of the $D$ and $A$ coefficients. For the basis vectors we have

$$
\chi^{(n)} \sim a^{-m-n+1 / 2}, \quad \Psi^{(n)} \sim a^{-1 / 2} .
$$

By exploiting again the known scaling behaviour (4.9) of $W_{g}(p)$, one can just as in the symmetric case derive certain homogeneity conditions that the $A$ and $D$ coefficients must fulfill. One finds

$$
A_{g}^{(n)}: \quad N_{M} \geq 2-2 g, \quad \sum_{j=1}^{s}\left(\alpha_{j}-1\right) \leq 3 g-n-1 ;
$$




$$
D_{g}^{(n)}: \quad N_{M} \geq 1-2 g, \quad \sum_{j=1}^{s}\left(\alpha_{j}-1\right) \leq 3 g-1 .
$$

The conditions are different for the $A$ and $D$ coefficients due to the different scaling behaviour of the basis vectors. Terms that are important in the double scaling limit are only those for which $s \leq m$ and for which the equality sign holds in both relations in (4.20) or both relations in (4.21). Comparing the second relation in (4.21) with (4.13) we see that all $D$-terms vanish in the double scaling limit. Furthermore, comparing (4.20) with (3.16) we see that all $J$ dependent $A$ terms disappear. Hence in the double scaling limit everything is expressed only in terms of the $M$ 's and $d$, and all terms have $\gamma=g-1$.

As is mentioned earlier, $D_{g}^{(n)}$ can be obtained from $A_{g}^{(n)}$ by performing the replacements $x \leftrightarrow y$ and $J \leftrightarrow M$ Hence we also have that the following homogeneity requirements should be fulfilled

$$
\begin{gathered}
A_{g}^{(n)}: \quad N_{J} \geq 1-2 g, \quad \sum_{j=1}^{l}\left(\beta_{j}-1\right) \leq 3 g-1 ; \\
D_{g}^{(n)}: \quad N_{J} \geq 2-2 g, \quad \sum_{j=1}^{l}\left(\beta_{j}-1\right) \leq 3 g-n-1 .
\end{gathered}
$$

These relations could of course be derived by analyzing the scaling behaviour assuming that the extra zeros accumulate at $y$. This implies fixing $x$ and replacing (4.14) and $(4.15)$ with

$$
\begin{aligned}
& p=y_{c}-a \pi, \\
& y=y_{c}+a \Lambda^{1 / m} .
\end{aligned}
$$

In this situation only terms for which $l \leq m$ and for which the equality sign holds in both relations in (4.22) or both relations in (4.23) will contribute in the double scaling limit. For symmetry reasons, now all $A$ terms plus $M$-dependent $D$ terms disappear. Also the free energy is expressed under these circumstances entirely in terms of the $J$ 's and $d$. We note that independently of the details of the prescription for taking the double scaling limit, in this limit the multi-loop correlator $W_{g}\left(p_{1}, \ldots, p_{s}\right)$ depends on at most $3 g-2+s$ moments and the free energy $F_{g}$ depends on at most $3 g-2$ moments.

\subsection{Determining $W_{g}(p)$ in the d.s.l.}

The homogeneity conditions derived in the previous sections allow us to determine whether a given term contributes to the double scaling limit or not. As an example, let us consider the case of a non-symmetric potential where the critical behaviour is associated with the endpoint $x$. Using $(4.20)$ we see that only $A_{2}^{(5)}$, the first term 
of $A_{2}^{(4)}$ and the first two terms of $A_{2}^{(3)}$ of the long list of complicated expressions for the $D$ and $A$ coefficients of $W_{2}(p)$ given in Section 3.2 survive in the double scaling limit. Obviously it would be convenient to have an algorithm which gives as output only terms which are relevant for the continuum limit. An algorithm which gives us all potentially relevant terms can be obtained by a slight modification of the one described in Section 3.2. By potentially relevant we mean relevant for $m$ multi-critical models with $m$ sufficiently large.

It is easy to convince oneself that terms which are relevant for the double scaling limit of $W_{g}(p)$ can only appear from terms which are relevant for the double scaling limit of the right hand side of the loop equation. Let us assume that we know the scaling relevant versions of $A_{G}^{(n)}$ and $D_{G}^{(n)}$ for $G=1, \ldots, g$ and let us assume that we want to calculate the double scaling limit of the right hand side of the loop equation for $W_{g+1}$. First of all we note that all terms in the basis vectors $\chi^{(n)}(p)$ and $\Psi^{(n)}(p)$ show the same scaling behaviour so none of them can be ignored. However we can replace all occurrences of $(p-y)$ with $\left(x_{c}-y\right)=d_{c}$ in this limit. It is known from earlier analysis [14] that for a $m$ multi-critical model $W_{g}(p, p)$ scales as

$$
W_{g}(\pi, \pi) \sim a^{-2 g(m+1 / 2)-2}
$$

Using equation (4.9) it is easy to show that all the products in the sum in (2.12) have the same scaling behaviour. Hence, no superfluous terms appear from the sum if we start from the double scaled versions of the $W_{G}, G=1, \ldots g$.

However, if we calculate $W_{g}(p, p)$ by letting the loop insertion operator as written in (3.26) act on $W_{g}(p)$, irrelevant terms will appear even if we start from double scaled version of $W_{g}(p)$. By comparing (4.9) and (4.26), we see that only operators in $d / d V(p)$ which lower the power of $a$ by $m+3 / 2$ give rise to the relevant terms. Therefore, we should discard all other operators. By examining carefully each term in (3.26), one finds that only the following part of the loop insertion operator remains in the double scaling limit:

$$
\frac{d}{d V(p)_{x}}=\sum_{n} \frac{d M_{n}}{d V(p)} \frac{\partial}{\partial M_{n}}+\frac{d x}{d V(p)} \frac{\partial}{\partial x} \quad \text { (d.s.l.) }
$$

where

$$
\begin{aligned}
& \frac{d M_{n}}{d V(p)}=-(n+1 / 2)\left\{\Phi_{x}^{(n+1)}(p)-\frac{M_{n+1}}{M_{1}} \Phi_{x}^{(1)}(p)\right\} \quad(\text { d.s.l. }) \\
& \frac{d x}{d V(p)}=\frac{1}{M_{1}} \Phi_{x}^{1}(p) \quad(\text { d.s.l. })
\end{aligned}
$$

and

$$
\Phi_{x}^{(n)}(p)=(p-x)^{-n}\left\{d_{c}(p-x)\right\}^{-1 / 2} \quad \text { (d.s.l.). }
$$


We are now in a position to calculate the double scaling limit of the right hand side of the loop equation for $W_{g+1}$ provided we know the double scaled versions of $W_{1}(p), \ldots, W_{g}(p)$. We see that, since all the $y$-dependence has disappeared, we do not have to perform any decomposition of the result. This is in accordance with the outcome of the analysis of the previous section that all $D$ terms disappear in the double scaling limit. There it was also found that all $J$-dependent $A$ terms would vanish. This also appears from the formulas above. No $J$ terms ever appear if we do not start out with any and we do not.

The starting point of the iteration procedure is of course as before $W_{0}(p, p)$ but now we should take only the part of it that contributes in the double scaling limit. From equation (3.23) we find 5

$$
W_{0}^{(N S)}(p, p)=\frac{1}{16} \frac{1}{(p-x)^{2}} \quad(\text { d.s.l. }) .
$$

There is an interesting feature of the $A$ coefficients. For all genera it holds that $A_{g}^{(1)}=$ $A_{g}^{(2)}=0$. This is because the smallest negative power of $(p-x)$ appearing in any basis vector is $3 / 2$. By multiplication of two basis vectors or by application of the loop insertion operator this power will be lowered by at least $3 / 2$. Hence terms of the type $(p-x)^{-1}$ and $(p-x)^{-2}$ never turn up on the right hand side of the loop equation.

We have calculated the $A$ coefficients as they look in the double scaling limit for $g=2,3$ and 4 following the iterative procedure outlined above. The results for $g=2$ and $g=3$ read

$$
\begin{aligned}
A_{2}^{(1)}= & 0 \\
A_{2}^{(2)}= & 0, \\
A_{2}^{(3)}= & \frac{49 M_{2}{ }^{2}}{256 d_{c} M_{1}{ }^{4}}-\frac{5 M_{3}}{32 d_{c} M_{1}{ }^{3}}, \\
A_{2}^{(4)}= & \frac{-49 M_{2}}{128 d_{c} M_{1}{ }^{3}}, \\
A_{2}^{(5)}= & \frac{105}{256 d_{c} M_{1}{ }^{2}}, \\
A_{3}^{(1)}= & 0, \\
A_{3}^{(2)}= & 0, \\
A_{3}^{(3)}= & \frac{-5355 M_{2}{ }^{5}}{512 d_{c}^{2} M_{1}{ }^{9}}+\frac{7995 M_{2}{ }^{3} M_{3}}{256 d_{c}^{2} M_{1}{ }^{8}}-\frac{32845 M_{2} M_{3}{ }^{2}+35588 M_{2}{ }^{2} M_{4}}{2048 d_{c}^{2} M_{1}{ }^{7}} \\
& +\frac{21\left(680 M_{3} M_{4}+773 M_{2} M_{5}\right)}{2048 d_{c}^{2} M_{1}{ }^{6}}-\frac{1155 M_{6}}{512 d_{c}^{2} M_{1}{ }^{5}},
\end{aligned}
$$

\footnotetext{
${ }^{5}$ Here and in the following we use the notation that the superscript $(S)$ refers to the case of the symmetric potential and the superscript $(N S)$ to the case of the non-symmetric potential (where the critical behaviour is associated with the endpoint $x$ ).
} 


$$
\begin{aligned}
A_{3}^{(4)}= & \frac{5355 M_{2}^{4}}{256 d_{c}^{2} M_{1}{ }^{8}}-\frac{21837 M_{2}^{2} M_{3}}{512 d_{c}^{2} M_{1}{ }^{7}} \\
& +\frac{17545 M_{3}^{2}+37373 M_{2} M_{4}}{2048 d_{c}^{2} M_{1}{ }^{6}}-\frac{10185 M_{5}}{2048 d_{c}^{2} M_{1}{ }^{5}}, \\
A_{3}^{(5)}= & \frac{-7371 M_{2}^{3}}{256 d_{c}^{2} M_{1}^{7}}+\frac{69373 M_{2} M_{3}}{2048 d_{c}^{2} M_{1}{ }^{6}}-\frac{17465 M_{4}}{2048 d_{c}^{2} M_{1}{ }^{5}}, \\
A_{3}^{(6)}= & \frac{64295 M_{2}^{2}}{2048 d_{c}^{2} M_{1}{ }^{6}}-\frac{30305 M_{3}}{2048 d_{c}^{2} M_{1}{ }^{5}}, \\
A_{3}^{(7)}= & \frac{-13013 M_{2}}{512 d_{c}^{2} M_{1}^{5}}, \\
A_{3}^{(8)}= & \frac{25025}{2048 d_{c}^{2} M_{1}^{4}} .
\end{aligned}
$$

We remind the reader that the terms listed above are only potentially relevant. Whether or not they are actually relevant depends on which multi-critical model one wants to consider. For a $m^{\prime}$ th multi-critical model all terms involving $M_{k}, k>m$ vanish in the double scaling limit. This is true whether one uses a minimal potential or not. We also remind the reader that we assumed that we had a non-symmetric potential and that the critical behaviour was associated with the endpoint $x$. In the case where the critical behaviour is associated with the endpoint $y$ all the formulas in this section still hold provided $d_{c}$ is replaced with $-d_{c}, x$ with $y$ and $M$ with $J$.

Let us turn now to the case of the symmetric potential. In view of the scaling relations (4.1) and (4.2) it might seem unnatural to work with terms like $(p-x)$ and $(p-y)$ and we will see below that there actually exists a way of avoiding this. However, for the moment we will analyze the scaling behaviour in the $x, y$ formalism by (formally) scaling $p$ to $-\sqrt{z_{c}}$ whenever it occurs in a term like $(p-y)$ and to $+\sqrt{z_{c}}$ whenever it occurs in a term like $(p-x)$. Now let us assume as in the non-symmetric case that we know the scaling relevant versions of $A_{G}^{(n)}$ and $D_{G}^{(n)}$ for $G=1, \ldots, g$ and that we want to calculate the double scaling limit of the right hand side of the loop equation for $W_{g+1}(p)$. Of course we still have that all terms in the basis vectors show the same scaling behaviour so we still have to keep all of them. However it is easy to convince oneself that in the double scaling limit all occurrences of $(p-y)$ in $\chi^{(n)}(p)$ can be replaced by $d_{c}$ and all occurrences of $(p-x)$ in $\Psi^{(n)}$ can be replaced by $-d_{c}$. Furthermore, it appears that when we calculate the sum of products on the right hand side of the loop equation all products that mix $\chi$ 's and $\Psi$ 's will be subdominant in the double scaling limit. These mixed products should hence be discarded. Finally to ensure the survival of only double scaling relevant terms in $W_{g}(p, p)$, the loop insertion operator should be written as

$$
\frac{d}{d V(p)}=\frac{d}{d V(p)_{x}}+\frac{d}{d V(p)_{y}}
$$


where $d / d V(p)_{y}$ can be obtained from $d / d V(p)_{x}$ given in equation (4.27) - 4.30) by replacing $x$ by $y, M$ by $J$ and $d_{c}$ by $-d_{c}$.

The starting point for the iterative procedure is of course the double scaled version of $W_{0}(p, p)$ which here reads

$$
W_{0}^{(S)}(p, p)=\frac{1}{16} \frac{1}{(p-x)^{2}}+\frac{1}{16} \frac{1}{(p-y)^{2}} \quad \text { (d.s.l.). }
$$

We see that the loop equation actually decouples into two independent equations. Each of these is a double scaled version of the loop equation for the non symmetric potential. One corresponds to the case where the critical behaviour is associated with the endpoint $x$, the other to the case where the critical behaviour is associated with the endpoint $y$. The $A_{g}^{(n)}$ 's are hence equal to those obtained in the non-symmetric case where the critical behaviour is associated with the endpoint $x$ and $D_{g}^{(n)}=(-1)^{n} A_{g}^{(n)}$. This means that the right hand side of the loop equation of the loop equation can be written as

$$
\text { the r.h.s. }=\sum_{n=1}^{3 g-1} d_{c}^{n} A_{g}^{(n)} \frac{1}{\left(p^{2}-z\right)^{n}}
$$

which seems to be the natural way of expressing it bearing in mind the scaling relations (4.1) and (4.2).

As a consequence, it becomes also more natural to express the generating functional as

$$
W_{g}^{(S)}(p)=\sum_{n=1}^{3 g-1} \tilde{A}_{g}^{(n)} \tilde{\chi}^{(n)}(p)
$$

where $\tilde{A}_{g}^{(n)}=A_{g}^{(n)} d_{c}^{n}$ and where we now have only one set of basis vectors $\tilde{\chi}^{(n)}(p)$ which fulfill

$$
\left\{\hat{K}-2 W_{0}(p)\right\} \tilde{\chi}^{(n)}(p)=\frac{1}{\left(p^{2}-z\right)^{n}} .
$$

It is easy to show that $\tilde{\chi}^{(n)}(p)$ should be chosen as

$$
\tilde{\chi}^{(n)}(p)=\frac{1}{M_{1}}\left\{\Phi_{z}^{(n)}(p)-\sum_{k=1}^{n-1} \tilde{\chi}^{(k)}(p) M_{n-k+1} d_{c}^{k-n}\right\}
$$

where

$$
\Phi_{z}^{(n)}(p)=\frac{1}{\left(p^{2}-z\right)^{n+1 / 2}} .
$$

Furthermore, one can easily convince oneself that the right hand side of the loop equation can be obtained directly in the form (4.35) if one replaces $(p-x)^{-1}$ by $d_{c}\left(p^{2}-z\right)^{-1}$ in the formulas $(4.27)-(4.30)$, i.e. if one carries out the iteration process starting from

$$
W_{0}^{S}(p, p)=\frac{d_{c}^{2}}{16\left(p^{2}-z\right)^{2}} \quad(\text { d.s.l. })
$$


and using the following expression for the loop insertion operator.

$$
\frac{d}{d V_{s}(p)}=\sum_{n} \frac{d M_{n}}{d V(p)} \frac{\partial}{\partial M_{n}}+\frac{d z}{d V(p)} \frac{\partial}{\partial z} \quad \text { (d.s.l.) }
$$

where

$$
\begin{aligned}
& \frac{d M_{n}}{d V(p)}=-(n+1 / 2)\left\{\Phi_{z}^{(n+1)}(p) d_{c}^{n}-\frac{M_{n+1}}{M_{1}} \Phi_{z}^{(1)}(p)\right\} d_{c} \quad \text { (d.s.l.) }, \\
& \frac{d z}{d V(p)}=\frac{d_{c}}{M_{1}} \Phi_{z}^{(1)}(p) \quad(\text { d.s.l. }) .
\end{aligned}
$$

This observation allows us t show explicitly that the hermitian and the complex matrix model are equivalent in the double scaling limit.

\subsection{Determining $F_{g}$ in the double scaling limit}

The starting point of the calculation is the double scaled version of $W_{g}(p)$ obtained as described in the previous section - and the strategy is again to rewrite the basis vectors in a form which allows one to identify $W_{g}(p)$ as a total derivative. However this time the rewriting in the case of $\chi^{(n)}$ is made with the aid of (4.28) instead of (3.27) and reads

$$
\chi^{(n)}(p)=\frac{1}{M_{1}}\left\{\frac{-2}{2 n-1} \frac{d M_{n-1}}{d V(p)}-\sum_{k=2}^{n-1} \chi^{(k)}(p) M_{n-k+1}\right\} \quad n \geq 2 .
$$

We do not need any expression for $\chi^{(1)}(p)$ since the sum in (4.44) starts at $k=2$ and since we know that in the double scaling limit $A_{g}^{(1)}=0$, neither we do need any expression for $\Phi_{x}(p)$. The relevant rewriting of $\Psi^{(n)}(p)$ appears from (4.44) when $M$ is replaced with $J$.

For the non-symmetric potential where the critical behaviour is associated with the endpoint $x$, we immediately find for genus one

$$
F_{1}^{(N S)}=-\frac{1}{24} \ln M_{1} \quad(\text { d.s.l. })
$$

This expression can of course alternatively be obtained by taking the double scaling limit of Eq. (3.33).

Before presenting the explicit results for $F_{g}^{(N S)}$ in genus 2, 3 and 4, let us describe the general structure of $F_{g}^{(N S)}$ as it appears from our iterative solution of the loop equation. We have

$$
F_{g}^{(N S)}=\sum_{\alpha_{j}>1}\left\langle\alpha_{1} \ldots \alpha_{s} \mid \alpha, \gamma\right\rangle_{g}^{h e r m} \frac{M_{\alpha_{1}} \ldots M_{\alpha_{s}}}{M_{1}^{\alpha} d^{\gamma}} \quad \text { (d.s.l.) } \quad g \geq 1
$$


where the rational numbers denoted by the brackets are identical to those of Eq. (3.1) having the same indices. The $\alpha_{j}$ 's obey Eq. (4.18), $\gamma$ is given by Eq. (4.8) and

$$
\alpha=2 g-2+s
$$

as it follows from Eq. (4.17).

Let us consider the $g=2$ case as an example of what structures are encoded by Eq. (4.46). For $g=2$ we have $\alpha_{j} \in[2,3 g-2]=[2,4]$ and the relation (4.18) gives

$$
\sum_{j=1}^{s}\left(\alpha_{j}-1\right)=3 g-3=3 \quad g=2
$$

so that $s \in[1,3 g-3]=[1,3]$. The restriction (4.48) admits $s=3$ and $\alpha_{1}=\alpha_{2}=\alpha_{3}=$ $2, s=2$ and $\alpha_{1}, \alpha_{2}=2,3$ and $s=1$ and $\alpha_{1}=4$. The proper powers of $M_{1}$ in the denominator for such terms are given by $\alpha=2 g-2+s=2+s$. The outcome of the iteration process is

$$
F_{2}^{(N S)}=\frac{-21 M_{2}{ }^{3}}{160 d_{c} M_{1}{ }^{5}}+\frac{29 M_{2} M_{3}}{128 d_{c} M_{1}{ }^{4}}-\frac{35 M_{4}}{384 d_{c} M_{1}{ }^{3}} .
$$

We note that all allowed values of $s$ and $\alpha_{i}$ 's actually appear. By taking a closer look at the loop insertion operator and the basis vectors it is easy to convince oneself that this will be the case for all genera. The expression (4.49) could of course also have been obtained by taking the double scaling limit of (3.37) following the recipe given in (4.17) and (4.18). We see that the expression for $F_{2}$ simplifies drastically in the double scaling limit.

The results for $g=3$ (where $s \leq 6$ ) and $g=4$ (where $s \leq 9$ ) read

$$
\begin{aligned}
F_{3}^{(N S)}= & \frac{2205 M_{2}{ }^{6}}{256 d_{c}^{2} M_{1}{ }^{10}}-\frac{8685 M_{2}^{4} M_{3}}{256 d_{c}^{2} M_{1}{ }^{9}}+\frac{15375 M_{2}^{2} M_{3}^{2}}{512 d_{c}^{2} M_{1}{ }^{2}}+\frac{5565 M_{2}{ }^{3} M_{4}}{256 d_{c}^{2} M_{1}{ }^{8}} \\
& -\frac{5605 M_{2} M_{3} M_{4}}{256 d_{c}^{2} M_{1}{ }^{7}}-\frac{72875 M_{3}{ }^{3}}{21504 d_{c}^{2} M_{1}{ }^{7}}-\frac{3213 M_{2}{ }^{2} M_{5}}{256 d_{c}^{2} M_{1}{ }^{7}}+\frac{2515 M_{3} M_{5}}{512 d_{c}^{2} M_{1}{ }^{6}} \\
& +\frac{21245 M_{4}{ }^{2}}{9216 d_{c}^{2} M_{1}{ }^{6}}+\frac{5929 M_{2} M_{6}}{1024 d_{c}^{2} M_{1}{ }^{6}}-\frac{5005 M_{7}}{3072 d_{c}^{2} M_{1}{ }^{5}}
\end{aligned}
$$

and

$$
\begin{aligned}
F_{4}^{(N S)}= & -\frac{21023793 M_{2}{ }^{9}}{10240 d_{c}^{3} M_{1}{ }^{15}}+\frac{12829887 M_{2}{ }^{7} M_{3}}{1024 d_{c}^{3} M_{1}{ }^{14}}-\frac{98342775 M_{2}{ }^{5} M_{3}{ }^{2}}{4096 d_{c}^{3} M_{1}{ }^{13}} \\
& -\frac{4456305 M_{2}{ }^{6} M_{4}}{512 d_{c}^{3} M_{1}{ }^{13}}+\frac{16200375 M_{2}{ }^{3} M_{3}{ }^{3}}{1024 d_{c}^{3} M_{1}{ }^{12}}+\frac{26413065 M_{2}{ }^{4} M_{3} M_{4}}{1024 d_{c}^{3} M_{1}{ }^{12}} \\
& +\frac{12093543 M_{2}{ }^{5} M_{5}}{2048 d_{c}^{3} M_{1}{ }^{12}}-\frac{83895625 M_{2} M_{3}{ }^{4}}{32768 d_{c}^{3} M_{1}{ }^{11}}-\frac{68294625 M_{2}{ }^{2} M_{3}{ }^{2} M_{4}}{4096 d_{c}^{3} M_{1}{ }^{11}}
\end{aligned}
$$




$$
\begin{aligned}
& -\frac{12367845 M_{2}{ }^{3} M_{4}{ }^{2}}{2048 d_{c}^{3} M_{1}{ }^{11}}-\frac{13024935 M_{2}{ }^{3} M_{3} M_{5}}{1024 d_{c}^{3} M_{1}{ }^{11}}-\frac{15411627 M_{2}{ }^{4} M_{6}}{4096 d_{c}^{3} M_{1}{ }^{11}} \\
& +\frac{32418925 M_{3}{ }^{3} M_{4}}{24576 d_{c}^{3} M_{1}{ }^{10}}+\frac{17562825 M_{2} M_{3} M_{4}{ }^{2}}{4096 d_{c}^{3} M_{1}{ }^{10}}+\frac{578655 M_{2} M_{3}{ }^{2} M_{5}}{128 d_{c}^{3} M_{1}{ }^{10}} \\
& +\frac{10050831 M_{2}{ }^{2} M_{4} M_{5}}{2048 d_{c}^{3} M_{1}{ }^{10}}+\frac{5472621 M_{2}{ }^{2} M_{3} M_{6}}{1024 d_{c}^{3} M_{1}{ }^{10}}+\frac{44207163 M_{2}{ }^{3} M_{7}}{20480 d_{c}^{3} M_{1}{ }^{10}} \\
& -\frac{1511055 M_{2} M_{5}^{2}}{2048 d_{c}^{3} M_{1}{ }^{9}}-\frac{7503125 M_{4}^{3}}{36864 d_{c}^{3} M_{1}{ }^{9}}-\frac{2642325 M_{3} M_{4} M_{5}}{2048 d_{c}^{3} M_{1}{ }^{9}} \\
& -\frac{11532675 M_{3}{ }^{2} M_{6}}{16384 d_{c}^{3} M_{1}{ }^{9}}-\frac{6242775 M_{2} M_{4} M_{6}}{4096 d_{c}^{3} M_{1}{ }^{9}}-\frac{6968247 M_{2} M_{3} M_{7}}{4096 d_{c}^{3} M_{1}{ }^{9}} \\
& -\frac{4297293 M_{2}{ }^{2} M_{8}}{4096 d_{c}^{3} M_{1}{ }^{9}}+\frac{12677665 M_{2} M_{9}}{32768 d_{c}^{3} M_{1}^{8}}+\frac{8437275 M_{5} M_{6}}{32768 d_{c}^{3} M_{1}{ }^{8}} \\
& +\frac{8913905 M_{4} M_{7}}{32768 d_{c}^{3} M_{1}^{8}}+\frac{10156575 M_{3} M_{8}}{32768 d_{c}^{3} M_{1}^{8}}-\frac{8083075 M_{10}}{98304 d_{c}^{3} M_{1}{ }^{7} .}
\end{aligned}
$$

Needless to say that the results for the case where the critical behaviour is associated with the endpoint $y$ can be obtained from these by performing the replacements $M \rightarrow$ $J, d_{c} \rightarrow-d_{c}$.

It is obvious from the discussion in Section 4.1 and formula (4.44) that independently of the details of the prescription for taking the double scaling limit, $F_{g}$ depends in this limit on at most $3 g-2$ moments. Again we stress that the terms listed above are only potentially relevant. For a $m$ 'th multi-critical model all terms involving $M_{k}$, $k>m$ vanish too. This is true whether one uses a minimal potential or not. It is interesting to note that for the symmetric potential we get a sum of two identical terms and hence

$$
\left.F_{g}^{(S)}=2 F_{g}^{(N S)} \quad \text { (d.s.l. }\right)
$$

which is a well known property of the double scaling limit for the hermitian matrix model [24]. It can be traced back to the fact that the loop equation for a symmetric potential decouples into two independent equations. Equation (4.52) is the reason why the partition function of 2D quantum gravity is defined as the square root of the double scaling limit of the partition function (2.1) in the case of a symmetric potential. There is no such square root in the non-symmetric case and the proper continuum partition function can be obtained just as the double scaling limit of (2.1).

Of course we have determined up till now only the coefficients, $F_{g}$, of the genus expansion of the free energy (Cf. Eq (2.11)). For a $m^{\prime}$ th multi-critical model the relevant expansion parameter in the double scaling limit is $N a^{m+1 / 2}$. If we define moments $\mu_{k}$ by (Cf. Eq (4.3))

$$
M_{k}=a^{m-k} \mu_{k}, \quad k \in[1, m]
$$


we get by replacing $M_{k}$ with $\mu_{k}$ for $k \in[1, m]$ and setting $M_{k}$ equal to zero for $k>m$ in the formulas above exactly the coefficient of the expansion in the double scaling parameter. A similar statement holds for the results of the previous section.

\subsection{A remark on the complex matrix model}

The complex matrix model is defined by the partition function

$$
Z^{C}=e^{N^{2} F^{C}}=\int_{N \times N} d \phi^{\dagger} d \phi \exp \left(-N V_{C}\left(\phi^{\dagger} \phi\right)\right)
$$

where the integration is over complex $N \times N$ matrices and

$$
V_{C}\left(\phi^{\dagger} \phi\right)=\sum_{j=1}^{\infty} \frac{g_{j}}{j} \operatorname{Tr}\left(\phi^{\dagger} \phi\right)^{j}
$$

Its generating functional is defined by

$$
W^{C}(p)=\frac{1}{N} \sum_{k=0}^{\infty}\left\langle\operatorname{Tr}\left(\phi^{\dagger} \phi\right)^{k}\right\rangle / p^{2 k+1}
$$

and the $s$-loop correlator $(s \geq 2)$ by

$$
\left.W^{C}\left(p_{1}, \ldots, p_{s}\right)=N^{s-2} \sum_{k_{1}, \ldots, k_{s}=1}^{\infty}\left\langle\operatorname{Tr}\left(\phi^{\dagger} \phi\right)^{k_{1}} \ldots \operatorname{Tr}\left(\phi^{\dagger} \phi\right)^{k_{s}}\right)\right\rangle_{c o n n .} / p_{1}^{2 k_{1}+1} \ldots p_{s}^{2 k_{s}+1}
$$

so that

$$
W^{C}\left(p_{1}, \ldots, p_{s}\right)=N^{s-2}\left\langle\operatorname{Tr} \frac{p_{1}}{p_{1}^{2}-\phi^{\dagger} \phi} \ldots \operatorname{Tr} \frac{p_{s}}{p_{s}^{2}-\phi^{\dagger} \phi}\right\rangle_{c o n n} .
$$

In Ref. [19] an iterative procedure which enables one to calculate $W_{g}^{C}\left(p_{1}, \ldots, p_{s}\right)$ for any genus $g$ and any $s$ starting from $W_{0}^{C}(p)$ was developed. This procedure is much the same as for the hermitian matrix model. It is based on the loop equation, the loop insertion operator and a suitable change of variables. In Ref. [19 the studies were carried out only away from the double scaling limit. In the Appendix we show how the iterative scheme for the complex matrix model can be modified to give directly the result in the double scaling limit. The modified scheme provides us with an exact proof that the hermitian and the complex matrix model are equivalent in the double scaling limite. We find that

$$
\begin{aligned}
W_{g}^{C}\left(p_{1}, \ldots, p_{s}\right) & =\frac{1}{4^{g+s-1}} W_{g}^{(S)}\left(p_{1}, \ldots, p_{s}\right) \quad(\text { d.s.l. }) \\
F_{g}^{C} & =\frac{1}{4^{g-1}} F_{g}^{(S)} \quad(\text { d.s.l. })
\end{aligned}
$$

\footnotetext{
${ }^{6}$ The fact that the hermitian and complex matrix models belong to the same universality class and that the correlators therefore coincide in the double scaling limit does not contradict Ref. 25] where it was shown that the discrete Virasoro operators for the complex matrix model do not have continuum limit.
} 
independently of the type of critical behaviour considered. In particular we see that the partition function of 2D quantum gravity can also be obtained as the square root of the double scaling limit of the partition function (4.54) provided the integration is over $N / 2 \times N / 2$ matrices in stead of over $N \times N$ matrices. This is of course in accordance with the fact that the number of independent components of a $N / 2 \times N / 2$ complex matrix is the same as that of a $N \times N$ hermitian matrix.

\section{$5 \quad$ Relation to moduli space}

The equivalence between the double scaling limit of the hermitian matrix model and the Kontsevich model has been proven in various ways in Refs. [26, 27]. We present in this section another proof directly in terms of the moments introduced above. For this purpose we describe a limiting procedure which allows us to obtain the Kontsevich model directly from the hermitian matrix model. We discuss also the connection between the hermitian matrix model and a certain discretization of moduli space.

\section{$5.1 \quad$ The limiting procedure}

As we have seen in Section 4.3, the double scaling limit of the free energy is given by the expansion (4.46) which encodes in a remarkable way all multi-critical behaviour of the hermitian matrix model. Since most of the terms originally present in the expression for $F_{g}$ vanish in the double scaling limit the question arises whether it is possible to formulate a matrix model which gives solely the coefficients which are relevant for the double scaling limit The answer to this question is - yes - and the proper model is known as the Kontsevich model.

The Kontsevich model is defined by the integral 99

$$
Z_{K}[M, n]=\frac{\int_{n \times n} d X \exp \left\{n \operatorname{tr}\left(-\frac{1}{2} M X^{2}+\frac{X^{3}}{6}\right)\right\}}{\int_{n \times n} d X \exp \left\{n \operatorname{tr}\left(-\frac{1}{2} M X^{2}\right)\right\}} .
$$

there $M=\operatorname{diag}\left(m_{1}, \ldots, m_{n}\right)$ (at first) is a positive definite matrix and the integration is over hermitian $n \times n$ matrices. The genus expansion of the free energy is given by

$$
\ln Z_{K}[M, n]=\sum_{g=0}^{\infty} n^{2-2 g} F_{g}^{K o n t} .
$$

The goal of this section is to show that the Kontsevich model can be obtained from the hermitian matrix model by a certain limiting procedure which looks conceptually different from the double scaling limit. We shall see however in Section 5.2 that this limiting procedure leads to a result which is identical to the one obtained in the double scaling limit. 
In order to formulate the limiting procedure let us recall some identities. Consider the hermitian matrix model as defined in Eq. (2.1) with $N=-\xi n$. following identity 18

$$
Z[g, N(\xi)]=\mathrm{e}^{-n \operatorname{tr} \Lambda^{2} / 2} Z_{P}[\Lambda, n], \quad N(\xi)=-\xi n
$$

where the partition function $Z_{P}[\Lambda, n]$ is associated with the Penner model [29], in the external field $\Lambda=\operatorname{diag}\left(\lambda_{1}, \ldots, \lambda_{n}\right)$ :

$$
Z_{P}[\Lambda, n]=\int_{n \times n} d X \exp \left[n \operatorname{tr}\left(\Lambda X-\frac{1}{2} X^{2}-\xi \log X\right)\right] .
$$

The identification is valid provided the set of coupling constants $\{g\}=\left\{g_{0}, g_{1}, \ldots\right\}$ and the matrix $\Lambda$ are related by the Miwa transformation

$$
g_{k}=\frac{1}{n} \operatorname{tr} \Lambda^{-k}-\delta_{k 2} \quad k \geq 1, \quad g_{0}=\frac{1}{n} \operatorname{tr} \log \Lambda^{-1} .
$$

In order to show how the partition function (5.1) can be obtained from (5.4), we note that $Z_{P}[\Lambda, n]$ can be reformulated as the so-called Kontsevich-Penner model 30]:

$$
Z_{K P}[\eta, n]=\frac{\int_{n \times n} d X \exp \left\{-\xi n \operatorname{tr}\left[\frac{1}{2} \eta X \eta X+(\log (1-X)+X)\right]\right\}}{\int_{n \times n} d X \exp \left\{-\xi n \operatorname{tr}\left[\frac{1}{2} \eta X \eta X-\frac{1}{2} X^{2}\right]\right\}}
$$

provided

$$
\Lambda=\sqrt{\xi}\left(\eta+\eta^{-1}\right)
$$

We note in addition that the model (5.6) possesses a remarkable connection to a discretization of moduli space which will be discussed in Section 5.4. Here we will be interested in the connection to the usual continuum moduli space, i.e. to the Kontsevich model which precisely has the interpretation as the generating functional for intersection indices on moduli space (Cf. to Section 5.3). In order to highlight this connection we choose a specific parametrization of $\eta$ and $\xi$ :

$$
\eta=\mathrm{e}^{\varepsilon m}, \quad \xi=\frac{1}{2 \varepsilon^{3}}
$$

where $m$ is to be identified with the matrix, $M$ which appears in the definition (5.1) of the Kontsevich model, and where $\varepsilon$ is presently just an expansion parameter which

\footnotetext{
${ }^{7}$ We should point out a subtlety related to the representations (5.4) and (5.6). We have used a constant $\xi$ in these equations which has the opposite sign of that conventionally used. The "analytic" continuation of $\xi$ is needed in order to make contact with the Kontsevich model. We will not to try to "justify" it, since the idea is to provide some heuristic explanation of the observed identity of the expansion coefficients of the hermitian matrix model and the Kontsevich model.

${ }^{8}$ See also Ref. [28] where the proof was extended to finite $n$.
} 
we are going to take to zero, but which in Section 5.4 will be given the interpretation of the step of discretization of moduli space.

It is now trivial to check that we in the limit $\varepsilon \rightarrow 0$ reproduce (5.1) from (5.6) after rescaling $X \rightarrow \varepsilon X$. In this way we have managed to move continuously from $Z[g, N(\xi)]$ to $Z_{K}[m, n]$. Note that there is no need to take the limit $n \rightarrow \infty$ during these steps, but the size $N(\xi)=-\xi n$ of the original hermitian matrix goes to infinity as $\varepsilon \rightarrow 0$.

It is instructive to illustrate the proof just given by taking the $\varepsilon \rightarrow 0$ limit of the explicit formulas governing the hermitian matrix model. The basic equations (2.14) which in the original matrix model determined the endpoints $x$ and $y$ in terms of the coupling constants $g_{i}$ have an equivalent formulation in terms of the eigenvalues $\lambda_{j}$ of $\Lambda$ when we rewrite the matrix model in terms of the Penner model as in (5.3). They are obtained by inserting in (2.14) the following relation between the eigenvalues $\lambda_{j}$ and the matrix model potential, $V$ :

$$
V^{\prime}(\omega)=\frac{1}{n} \sum_{j=1}^{n} \frac{1}{\lambda_{j}-\omega}-\omega .
$$

This relation follows directly from (5.5). By the use of (5.9) the equation (2.14) reads

$$
\begin{gathered}
\frac{1}{n} \sum_{i=1}^{n} \frac{1}{\sqrt{\left(\lambda_{i}-x\right)\left(\lambda_{i}-y\right)}}-\frac{x+y}{2}=0, \\
\frac{1}{n} \sum_{i=1}^{n} \frac{\lambda_{i}-\frac{x+y}{2}}{\sqrt{\left(\lambda_{i}-x\right)\left(\lambda_{i}-y\right)}}-\frac{(x-y)^{2}}{8}=-2 \xi .
\end{gathered}
$$

Notice that the normalization factor " 2 " in (2.14) has been changed to $-2 \xi$, since the size of the matrix is $N=-\xi n$.

Let us now apply the limiting procedure dictated by (5.8) to Eqs. (5.10) and (5.11):

$$
\Lambda=\sqrt{\xi}\left(\mathrm{e}^{\varepsilon m}+\mathrm{e}^{-\varepsilon m}\right)=\frac{\sqrt{2}}{\varepsilon^{3 / 2}}+\sqrt{\frac{\varepsilon}{2}} m^{2}+O\left(\varepsilon^{5 / 2}\right) .
$$

In order to solve equations (5.10), (5.11) in terms of $x$ and $y$ we choose

$$
y=-\frac{\sqrt{2}}{\varepsilon^{3 / 2}}, \quad x=\frac{\sqrt{2}}{\varepsilon^{3 / 2}}+\sqrt{2} \varepsilon^{1 / 2} u_{0}+\ldots .
$$

From Eq. (5.10) we have the equation

$$
\frac{1}{n} \sum_{j=1}^{n} \frac{1}{\sqrt{m_{j}^{2}-2 u_{0}}}=u_{0}
$$

which appears multiplied by $\sqrt{\varepsilon}$. After the substitution into Eq. (5.11), we get that the leading term of order $1 / \varepsilon^{3}$ coming from $2 \xi$ and $(x-y)^{2} / 8$ cancels. It is crucial for this 
cancellation that $\xi>0$. The next order terms which are proportional to $1 / \varepsilon$ combine again into Eq. (5.14), which we recognize as nothing but the stationary condition [31] appearing in the Kontsevich model.

Let us now demonstrate how the 1-loop average of the hermitian matrix model is related to the one of the Kontsevich model. We start from genus zero inserting Eq. (5.9) into (2.13) which gives 18

$$
W_{0}(p)=\frac{1}{2}\left[\sqrt{(p-x)(p-y)}-p+\frac{1}{n} \sum_{j} \frac{1}{p-\lambda_{j}}\left(\frac{\sqrt{(p-x)(p-y)}}{\sqrt{\left(\lambda_{j}-x\right)\left(\lambda_{j}-y\right)}}-1\right)\right] .
$$

Eq. (5.10) ensures the vanishing of the $\mathcal{O}(1)$ term of the $1 / p$ expansion of the right hand side, while Eq. (5.11) implies that the $\mathcal{O}\left(p^{-1}\right)$ term equals $-\xi / p$. Contrary the representation (2.21) can be obtained from (5.15) by expanding in $1 / \lambda_{j}$ and using Eqs. (5.5) and (5.9).

To obtain an analogue of Eq. (5.12) for $p$, let us point out that the relation (5.5) implies

$$
\frac{\partial}{\partial \lambda_{i}}=-\frac{1}{\lambda_{i}} \frac{\partial}{\partial g_{0}}-\sum_{k=1}^{\infty} \frac{k}{\lambda_{i}^{k+1}} \frac{\partial}{\partial g_{k}} \quad n \rightarrow \infty
$$

which requires $n \rightarrow \infty$ in order for $g_{k}$ 's to be independent variables. Comparing with Eq. (2.8) one gets

$$
\frac{d}{d V(p)}=\left.\frac{\partial}{\partial \lambda_{i}}\right|_{\lambda_{i}=p} \quad n \rightarrow \infty
$$

Therefore we substitute

$$
p=\frac{\sqrt{2}}{\varepsilon^{3 / 2}}+\sqrt{\frac{\varepsilon}{2}} \pi+O\left(\varepsilon^{5 / 2}\right)
$$

where $\pi$ is to be understood as the momentum variable for the Kontsevich model.

Substituting (5.12), (5.13) and (5.18) into Eq. (5.15), one gets

$$
W_{0}(p)+\frac{p}{2}+\frac{1}{2 n} \sum_{j} \frac{1}{p-\lambda_{j}} \rightarrow \sqrt{\frac{2}{\varepsilon}}\left[W_{0}^{\text {kont }}(\pi)+\frac{\sqrt{\pi}}{2}+\frac{1}{2 n} \sum_{k} \frac{m_{k}}{\sqrt{\pi}} \frac{1}{\pi-m_{k}^{2}}\right]
$$

where

$$
\begin{aligned}
W_{0}^{\text {kont }}(\pi) & =\left.\frac{\partial}{\partial m_{i}^{2}}\right|_{m_{i}^{2}=\pi} \ln Z_{0}^{K o n t}[M, n] \\
& =\frac{1}{2}\left[\sqrt{\pi-2 u_{0}}-\sqrt{\pi}+\frac{1}{n} \sum_{j} \frac{1}{\pi-m_{j}^{2}}\left(\frac{\sqrt{\pi-2 u_{0}}}{\sqrt{m_{j}^{2}-2 u_{0}}}-\frac{m_{j}}{\sqrt{\pi}}\right)\right]
\end{aligned}
$$

We see that the subtraction needed to make contact with the Kontsevich model is exactly the same as the usual double scaling subtraction. For the two-loop correlator we have

$$
W_{0}(p, q)+\frac{1}{2} \frac{1}{(p-q)^{2}} \rightarrow\left(\frac{2}{\epsilon}\right)\left[W_{0}^{\text {Kont }}\left(\pi_{1}, \pi_{2}\right)+\frac{\frac{1}{2}\left(\pi_{1}+\pi_{2}\right)}{2\left(\pi_{1}-\pi_{2}\right)^{2} \sqrt{\pi_{1} \pi_{2}}}\right]
$$


We recognize the term $1 / 2(p-q)^{2}$ as being the non universal part of the two-loop correlator. Hence the renormalization needed in this case is also the usual one of the double scaling limit. For $p=q$ we find

$$
W_{0}(p, p) \rightarrow\left(\frac{2}{\epsilon}\right)\left[W_{0}^{\text {Kont }}(\pi, \pi)+\frac{1}{16 \pi^{2}}\right]
$$

The appearance of the term $1 / 16 \pi^{2}$ when one wants to make contact with continuum physics is well known from the study of the double scaling limit of the Virasoro constraints for the hermitian matrix model [25]. It is evident from equation (5.21) that for the higher loop correlators it holds that

$$
W_{0}\left(p_{1}, \ldots, p_{s}\right) \rightarrow\left(\frac{2}{\epsilon}\right)^{s / 2} W_{0}^{K o n t}\left(\pi_{1}, \ldots, \pi_{s}\right), \quad s>2
$$

This multiplicative renormalization is also in compliance with the renormalization of the double scaling limit. The origin of the factors $(2 / \epsilon)^{1 / 2}$ is easy to understand bearing in mind the relations (5.12) and (5.17). In the next section we show that the limiting procedure described above actually leaves us with the same terms as the double scaling limit does. It is worth noting that this means that the Kontsevich correlators encode information about continuum correlators at all multi-critical points. This information is even easily accessible. To obtain the continuum correlators one rewrites first Eq. (5.14) as the genus zero string equation

$$
\sum_{k=0}^{\infty} \frac{(2 k-1) ! !}{k !} t_{k} u_{0}^{k}=0
$$

where

$$
t_{k}=\frac{1}{n} \operatorname{tr} M^{-2 k-1}-\delta_{k 1} \quad k \geq 0
$$

are continuum coupling constants which all vanish at the $m^{\text {th }}$ multi-critical point except for $t_{m}$ and $t_{0}$ with the latter playing the role of the cosmological constant. The continuum correlators can hence be found by expanding the Kontsevich correlators in powers of $1 / m_{j}$. Equation (5.14) ensures the vanishing of the $1 / \sqrt{\pi}$ term in the the expansion of the right hand side of (5.20).

We are now ready to present one more proof of the fact that by carrying our limiting procedure for the hermitian matrix model we recover the Kontsevich model. For this aim let us consider the Schwinger-Dyson equation of the model (5.4) which reads [30]

$$
\left.\frac{1}{n^{2}} \frac{\partial \tilde{W}\left(\lambda_{i}\right)}{\partial \lambda_{j}}\right|_{\lambda_{j}=\lambda_{i}}+\left(\tilde{W}\left(\lambda_{i}\right)\right)^{2}+\frac{1}{n} \sum_{j \neq i} \frac{\tilde{W}\left(\lambda_{j}\right)}{\lambda_{j}-\lambda_{i}}=\frac{\lambda_{i}^{2}}{4}-\xi+\frac{1}{2}
$$

where

$$
\tilde{W}\left(\lambda_{i}\right)=W\left(\lambda_{i}\right)+\frac{\lambda_{i}}{2}+\frac{1}{2 n} \sum_{j \neq i} \frac{1}{\lambda_{i}-\lambda_{j}}
$$


Eq. (5.26) can be obtained by inserting Eq. (5.9) into the loop equation (2.12).

Substituting Eqs. (5.12), (5.20) and (5.22) and assuming multiplicative renormalizability of the higher genera contributions to the correlators (as in the double scaling limit) one gets from equation (5.26) the following equation

$$
\left.\frac{1}{n^{2}} \frac{\partial \tilde{W}^{k o n t}\left(m_{i}^{2}\right)}{\partial m_{j}^{2}}\right|_{m_{j}^{2}=m_{i}^{2}}+\left(\tilde{W}^{k o n t}\left(m_{i}^{2}\right)\right)^{2}+\frac{1}{n} \sum_{j \neq i} \frac{\tilde{W}^{k o n t}\left(m_{j}^{2}\right)}{m_{j}^{2}-m_{i}^{2}}=\frac{m_{i}^{2}}{4}
$$

where

$$
\tilde{W}^{\text {kont }}\left(m_{i}^{2}\right)=W^{\text {kont }}\left(m_{i}^{2}\right)+\frac{m_{i}}{2}+\frac{1}{2 n} \sum_{j \neq i} \frac{m_{j}}{m_{i}}\left(\frac{1}{m_{i}^{2}-m_{j}^{2}}\right)
$$

This is exactly the Schwinger-Dyson equation of the Kontsevich model.

\subsection{The equivalence of the d.s.l. with Kontsevich model}

The equivalence of the Kontsevich model and the double scaling limit of the hermitian matrix model in genus zero was demonstrated in Ref. [31] by comparing explicit solutions. In order to compare next orders of the genus expansions, let us consider the following expression for $F_{g}^{K o n t}$ conjectured in Ref. [17] for the Kontsevich model:

$$
F_{g}^{K o n t}=\sum_{\alpha_{j}>1}\left\langle\alpha_{1} \ldots \alpha_{s} \mid \alpha\right\rangle_{g}^{k o n t} \frac{I_{\alpha_{1}} \cdots I_{\alpha_{s}}}{\left(I_{1}-1\right)^{\alpha}} \quad g \geq 1
$$

where the moments $I_{k}$ 's are defined byt:

$$
I_{k}(M)=\frac{1}{n} \sum_{j=1}^{n} \frac{1}{\left(m_{j}^{2}-2 u_{0}\right)^{k+1 / 2}} \quad k \geq 0
$$

and $u_{0}(M)$ is determined by the equation $u_{0}=I_{0}\left(u_{0}, M\right)$, i.e. by Eq. (5.14). When we compare the coefficients $\langle\cdot\rangle_{g}^{\text {kont }}$ calculated in Ref. [17] with the $\langle\cdot\rangle_{g}^{\text {herm }}$ calculated in the double scaling limit in Section 4.3, we see that they are identical. In this section we show that this is true to all orders in the genus.

For any value of $\varepsilon$ in (5.8) we have the expansion (3.1) of $F_{g}$ of the hermitian matrix model in terms of $M_{k}$ 's and $J_{k}$ 's. We are now going to show that the terms which survive in the limit $\varepsilon \rightarrow 0$ are the same as those that survive in the double scaling limit (4.46), and that the $M_{k}$ 's are related directly to the $I_{k}$ 's of the Kontsevich model defined by (5.31) and (5.14).

Let us now discuss the scaling behaviour of the moments $M_{k}$ and $J_{k}$. By substituting (5.9) into Eqs. (2.17) and (2.18), we obtain for the moments $M_{k}, J_{k}$ :

$$
M_{k}=\frac{1}{n} \sum_{j} \frac{1}{\left(\lambda_{j}-x\right)^{k+1 / 2}\left(\lambda_{j}-y\right)^{1 / 2}}-\delta_{k 1} \quad k \geq 1,
$$

\footnotetext{
${ }^{9}$ This definition differs by a factor $-(2 k-1)$ !! from the $I_{k}$ 's used in Ref. [17].
} 


$$
J_{k}=\frac{1}{n} \sum_{j} \frac{1}{\left(\lambda_{j}-x\right)^{1 / 2}\left(\lambda_{j}-y\right)^{k+1 / 2}}-\delta_{k 1} \quad k \geq 1 .
$$

From this representation and Eqs. (5.12), (5.13) it is easy to infer for the moments $M_{k}$ and $J_{k}$ and for the parameter $d$ the following scaling rules:

$$
\begin{aligned}
J_{k} & \rightarrow-2^{-(3 k / 2+1)} \varepsilon^{(3 k+1) / 2} I_{0}+\delta_{k 1}, \\
M_{k} & \rightarrow-2^{(k-1) / 2} \varepsilon^{-(k-1) / 2}\left(I_{k}-\delta_{k 1}\right), \\
d & \rightarrow 2^{3 / 2} \varepsilon^{-3 / 2}
\end{aligned}
$$

where $I_{k}$ 's are the the Kontsevich moments defined by (5.31), (5.14).

Let us turn to the general expression (3.1) for $F_{g}$. Then the power in $\varepsilon$ for a specific term

$$
\frac{M_{\alpha_{1}} \cdots M_{\alpha_{s}} J_{\beta_{i}} \cdots J_{\beta_{l}}}{d^{\gamma} M_{1}^{\alpha} J_{1}^{\beta}}
$$

is

$$
[\varepsilon]=\sum_{i=1}^{l} \frac{3 \beta_{i}+1}{2}-\sum_{j=1}^{s} \frac{\alpha_{j}-1}{2}+\frac{3}{2} \gamma .
$$

Clearly, due to the formulas $(3.6)$ and $(3.8)$, we find that $[\varepsilon] \geq 0$. $[\varepsilon]=0$ is possible if and only if $l=0, \gamma=g-1$ and

$$
\sum_{j=1}^{s}\left(\alpha_{j}-1\right)=3 g-3
$$

This means that we have precisely the same terms surviving as in the generic double scaling limit (4.46). By taking a closer look at the loop insertion operator (3.26) one finds that such terms always have $\beta=0$ and hence $N_{M}=2-2 g$. We therefore conclude that starting from the generic hermitian matrix model and letting $\varepsilon \rightarrow 0$ we will reproduce (5.30), (5.31) and (5.14) the coefficients in front of the $I_{k}$ terms being

those inherited from the hermitian matrix model. Hence we have $\langle\cdot\rangle_{g}^{\text {herm }}=\langle\cdot\rangle_{g}^{\text {Kont }}$ since we saw explicitly that the partition function $Z[g, N(\xi)]$ is identical to the partition function of the Kontsevich model in the limit $\varepsilon \rightarrow 0, \xi=1 / 2 \varepsilon^{3}$.

Thus the coefficients $\langle\cdot\rangle_{g}^{\text {herm }}$ which survive in the double scaling limit have an interpretation as intersection indices on moduli space, since this is the interpretation of the coefficients of the Kontsevich model. In Section 5.4 we are going to show that the additional coefficients which appear away from the double scaling limit can also be given a geometric interpretation on moduli space.

\subsection{Intersection indices on moduli space}

It has been known for some time that matrix models might be very useful in providing explicit realizations of the moduli spaces $\mathcal{M}_{g, s}$ of Riemann surfaces of genus $g$ and $s$ 
marked points $x_{1}, \ldots, x_{s}$. The first example is the Penner model [29], which allowed a relatively simple calculation of the virtual Euler characteristics of these spaces. The next example is the Kontsevich model, which allows a calculation of intersection indices on $\mathcal{M}_{g, s}$.

It is not our intention here to define or discuss these concepts in any detail. Let us only note, to fix the notation, that there exist $s$ natural line bundles $\mathcal{L}_{i}, i=1, \ldots, s$ on $\mathcal{M}_{g, s}$. The fiber of $\mathcal{L}_{i}$ at a point $\Sigma \in \mathcal{M}_{g, s}$ is the cotangent space to the point $x_{i}$, viewed as a point on the surface $\Sigma$. The line bundles have first Chern classes $c_{1}\left(\mathcal{L}_{i}\right)$, which can be represented by the curvature 2 -form of an arbitrary $U(1)$ connection on $\mathcal{L}_{i}$. If we have non-negative integers $\alpha_{i}$ such that

$$
\sum_{i=1}^{s} \alpha_{i}=\frac{1}{2} \text { real } \operatorname{dim} \mathcal{M}_{g, s}=3 g-3+s
$$

it is possible to form the integral [7]

$$
\left\langle\tau_{\alpha_{1}} \cdots \tau_{\alpha_{s}}\right\rangle=\int_{\overline{\mathcal{M}}_{g, s}} c_{1}\left(\mathcal{L}_{1}\right)^{\alpha_{1}} \cdots c_{1}\left(\mathcal{L}_{s}\right)^{\alpha_{s}}
$$

where products and powers are exterior products and powers and $\overline{\mathcal{M}}_{g, s}$ refers to a suitable compactification (the so-called Deligne-Mumford compactification) of $\mathcal{M}_{g, s}$.

The numbers $\left\langle\tau_{\alpha_{1}} \cdots \tau_{\alpha_{s}}\right\rangle$ are topological invariants and one of the achievements of Kontsevich was that he found [9] a more manageable representation by using a combinatorial decomposition of $\mathcal{M}_{g, s}$ inherited from the physicists "fat-graph" expansion of the hermitian matrix integrals. If we have a double-line graph with $s$ faces (dual to the $s$ punctures), we can assign a perimeter $p_{f}=\sum_{e} l_{e}$ to each face, $l_{e}$ being the length of the edge $e$ and the sum being over edges which constitute the face $f$. The set of such double-line graphs with assigned $l$ 's is a combinatorial model $\mathcal{M}_{g, s}^{\text {comb }}$ of moduli space. Cells $G$ have dimensions over the real numbers obtained by counting the number of independent $l_{e}$ 's for fixed $p_{f}$ 's. The result is that

$$
\text { real } \operatorname{dim} G \leq 2(3 g-3+s)
$$

where the equality sign is valid if and only if all vertices of the graph $G$ are trivalent.

For each face $f_{i}$ one can introduce the 2 -form $\omega_{i}$, which can be considered as the combinatorial version of $c_{1}\left(\mathcal{L}_{i}\right)$ related to the puncture $x_{i}$ :

$$
\omega_{i}=\sum_{a<b} d\left(\frac{l_{a}}{p_{f_{i}}}\right) \wedge d\left(\frac{l_{b}}{p_{f_{i}}}\right)
$$

where $l_{a}$ are the lengths of the edges of $f_{i}$, which are assumed to be cyclic ordered. For fixed $p_{f}$ 's we can now write the formula for intersection indices as

$$
\left\langle\tau_{\alpha_{1}} \cdots \tau_{\alpha_{s}}\right\rangle=\int \omega_{1}^{\alpha_{1}} \cdots \omega_{s}^{\alpha_{s}}
$$


provided the integral is over cells $G$ of maximal dimension, i.e. provided all vertices are trivalent. Let us define denote the complex dimension of $\mathcal{M}_{g, s}$ by $d_{g, s}$, i.e.

$$
d_{g, s}=3 g-3+s
$$

and introduce the 2-form

$$
\Omega=\sum_{i=1}^{s} p_{f_{i}}^{2} \omega_{i}
$$

It acts as a generating function for intersection indices:

$$
\int \Omega^{d_{g, s}}=\sum_{\alpha_{1}+\ldots+\alpha_{s}=d_{g, s}}\left\langle\tau_{\alpha_{1}} \cdots \tau_{\alpha_{s}}\right\rangle_{g} d_{g, s} ! \prod_{i=1}^{s} \frac{p_{f_{i}}^{2 \alpha_{i}}}{\alpha_{i} !}
$$

By a Laplace transformation we can trade the $p_{i}$ 's for $m_{i}$ 's by

$$
\int_{0}^{\infty} d p_{i} e^{-p_{i} m_{i}} p^{2 \alpha_{i}}=\left(2 \alpha_{i}\right) ! m_{i}^{-2 \alpha_{i}-1}
$$

and one of the main results of Kontsevich is that

$$
\sum_{\alpha_{1}+\ldots+\alpha_{s}=d_{g, s}}\left\langle\tau_{\alpha_{1}} \cdots \tau_{\alpha_{s}}\right\rangle_{g} \prod_{i=1}^{s} \frac{\left(2 \alpha_{i}-1\right) ! !}{m_{i}^{2 \alpha_{i}+1}}=\sum_{G} \frac{2^{-V}}{A u t(G)} \prod_{e \in G} \frac{2}{m_{f}+m_{f^{\prime}}}
$$

where the summation on the right hand side is over all double-line, trivalent, connected graphs $G$ of genus $g$ and with $s$ faces. The quantity denoted by $V$ is the number of vertices in $G$, e denote an edge in $G$ and $f$ and $f^{\prime}$ the faces sharing a given double-line e. Finally $\operatorname{Aut}(G)$ is the order of symmetry group of $G$. For future use we note that that the last factor can be written as

$$
\prod_{e \in G} \frac{1}{m_{f}+m_{f^{\prime}}}=\int_{0}^{\infty} \prod_{e \in G} d l_{e} e^{-l_{e}\left(m_{f}+m_{f^{\prime}}\right)}
$$

When we compare the right hand side of Eq. (5.49) with the definition of the Kontsevich integral (5.1) we see immediately that (5.49) represents the sum of the connected diagrams of genus $g$ and $s$ faces which would be generated by a perturbative expansion with respect to the gaussian part of the action. In the next section we shall extend this result to a more general interaction.

\subsection{The discretized moduli space}

As we shall see the results obtained in this article away from the critical regime is related to a representation of moduli space which incorporates explicitly the boundary components of the Deligne-Mumford compactification (or reduction) procedure. The basic ingredient in the new construction is a discretization of $\mathcal{M}_{g, s}^{c o m b}$. The length $l_{e}$ of an edge is assumed the belong to $\varepsilon Z_{+}$, where $\varepsilon$ is an expansion parameter, which 
eventually is going to be associated with the one in the Section 5.2. This means that the Laplace transform in $p_{i}$ performed in (5.48) is going to be replaced by a sum. The effect of this summation is heuristically described by referring to the representation (5.50):

$$
\int_{0}^{\infty} \prod_{e \in G} d l_{e} e^{-l_{e}\left(m_{f}+m_{f^{\prime}}\right)} \rightarrow \varepsilon^{3 d_{g, s}} \prod_{e \in G} \sum_{n_{e}=1}^{\infty} e^{-\varepsilon n_{e}\left(m_{f}+m_{f}^{\prime}\right)}=\varepsilon^{3 d_{g, s}} \prod_{(i j)} \frac{1}{e^{\varepsilon\left(m_{i}+m_{j}\right)}-1}
$$

where the power of $\varepsilon$ is valid for connected trivalent graphs. In the last product we recognize the propagator for the Kontsevich-Penner model (5.6) which involves the factor

$$
\frac{1}{\eta_{i} \eta_{j}-1}
$$

provided we make the assignment $\eta_{i}=\exp \left(\varepsilon m_{i}\right)$. This assignment is nothing but the one already made in (5.8), and the $\varepsilon$ assignment in (5.8) is also consistent with the $\varepsilon$ factor in (5.51). In order to fit our notations to the ones by Kontsevich it will be sometimes conveninent to use $\tilde{\xi}=2 \xi=\varepsilon^{-3}$. In fact the perturbative expansion of the Kontsevich-Penner model (5.6) can be written as

$$
\ln Z_{K P}[\eta, n]=\sum_{g=0}^{\infty} \sum_{s=0}^{\infty} n^{2-2 g}(\tilde{\xi})^{2-2 g-s} \sum_{G} \frac{2^{-V}}{A u t(G)} \prod_{(i j)} \frac{2}{\eta_{i} \eta_{j}-1}
$$

where the notation is as in (5.49), except that $\sum_{G}$ denotes the summation over all connected double-line graphs of genus $g$ and $s$ faces, not only the trivalent graphs as in (5.49). In this way we see that the Kontsevich-Penner model for finite $\varepsilon$ provides a generalization of the Kontsevich model in the sense that it in the limit $\varepsilon \rightarrow 0$ just reduces to the Kontsevich model, while it for $\varepsilon>0$ describes a discretized version of moduli space, which however allows us to access the boundary in more detail.

By use of this formalism it can be shown that the Kontsevich-Penner model allows an expansion, related to the Deligne-Mumford reduction in moduli space, which can be viewed as a generalization of the corresponding expansion of the Kontsevich model [20]. For the coefficient of $n^{2-2 g} \xi^{2-2 g-s}$ in the expansion (5.53) of $\ln Z_{K P}$ one has

$$
\begin{aligned}
F_{g, s}= & \varepsilon^{6 g-6+3 s} \sum_{\text {reductions }}(-1)^{r_{p}} \frac{\left(d_{g, s}-r_{p}\right) !}{d_{g, s} !} \\
& \times \prod_{j=1}^{p}\left[\sum_{\sum \alpha_{a}=d_{j}}\left\langle\tau_{\alpha_{1}} \ldots \tau_{\alpha_{f_{j}}} \tau_{0}^{(1)} \ldots \tau_{0}^{\left(k_{j}\right)}\right\rangle_{g_{j}} \frac{\left(f_{j}\right) !}{\left(f_{j}+k_{j}\right) !}\right. \\
& \times\left.\operatorname{tr} \prod_{k=1}^{f_{j}}\left(\frac{1}{\varepsilon} \frac{\partial}{\partial m_{k}}\right)^{2 \alpha_{k}} \frac{(-1)^{f_{j}}}{\prod_{k=1}^{f_{j}}\left(\mathrm{e}^{\varepsilon m_{k}}-1\right)}\right|_{\substack{\text { symmetrized } \\
\mathrm{e}^{\varepsilon m_{k \rightarrow-\mathrm{e}} \varepsilon m_{k}}}}
\end{aligned}
$$


As opposed to what was the case for the Kontsevich model one should now take into account all reductions of the original Riemann surface. The notation is as follows. For a given reduction, $p$ denotes the number of connected components of the reduced Riemann surface. These components are labeled by the index $j$. The $j$ 'th component has genus $g_{j}, f_{j}$ original punctures and $k_{j}$ punctures resulting from the reduction procedure. Note that there is no $m_{k}$ variables corresponding to these new insertions, that leads to appearing of additional symmetrical factors $\left(f_{j}\right) ! /\left(f_{j}+k_{j}\right)$ ! in (5.54). The corresponding moduli space has complex dimension $d_{j}=3 g_{j}-3+f_{j}+k_{j}$. The quantity $r_{p}$ is the power of reduction, defined by $\sum_{j=1}^{p} d_{j}=d-r_{p}$. The symmetrization ensures that $F_{g}$ is invariant under the replacements $M_{k} \rightarrow(-1)^{k+1} J_{k}$ as it must be.

The expression (5.54) resembles in many details the original answer by Kontsevich, the only two differences being that firstly the variables of expansion, which in the Kontsevich case appear in the combination $-\left(2 \alpha_{i}-1\right) ! ! \operatorname{tr} m^{-2 \alpha_{i}-1}$, in (5.54) appear in a more involved expression. Secondly the complete answer (5.54) contains information about the reductions of the Riemann surface.

Since, as shown explicitly in Section 5.1, the Kontsevich-Penner model can be mapped onto the general hermitian matrix model the information about this reduction is encoded in our expansion of the hermitian matrix model in terms of the moments $M_{k}, J_{k}$, and by exploring it we may hope to gain some information about the boundary of the moduli space. In order to do this, we need to expand moments $M_{k}, J_{k}$ and $d$ in terms of the somewhat unusual expansion parameters standing on the right hand side of Eq. (5.54). As the first step we need to resolve the constraint equations (2.14) in terms of these parameters. As the first approximation we have (in terms of eigenvalues):

$$
\begin{aligned}
M_{k} & \sim-\frac{1}{\sqrt{\xi}^{k+1}} \frac{1}{n} \sum_{i=1}^{n} \frac{\mathrm{e}^{\varepsilon m_{i}(k+1)}}{\left(1-\mathrm{e}^{\varepsilon m_{i}}\right)^{2 k+1}\left(1+\mathrm{e}^{\varepsilon m_{i}}\right)}+\delta_{k 1}, \\
J_{k} & \sim-\frac{1}{\sqrt{\xi}^{k+1}} \frac{1}{n} \sum_{i=1}^{n} \frac{\mathrm{e}^{\varepsilon m_{i}(k+1)}}{\left(1-\mathrm{e}^{\varepsilon m_{i}}\right)\left(1+\mathrm{e}^{\varepsilon m_{i}}\right)^{2 k+1}}+\delta_{k 1}, \\
d & \sim \sqrt{\xi}\left\{4-\frac{1}{\xi n} \sum_{i=1}^{n} \frac{2}{\left(\mathrm{e}^{\varepsilon m_{i}}-1\right)\left(1+\mathrm{e}^{\varepsilon m_{i}}\right)}\right\} .
\end{aligned}
$$

Let us now check the validity of the expansion (5.54), for the simplest non-trivial example, the moduli space $F_{1,1}$ (a torus with one marked point). Since $s=1$, we should extract only single trace terms from the formal expansion of the answer in product of traces. Using the explicit answer for $F_{1}$, we find that

$$
\begin{aligned}
-\left.\frac{1}{24} \ln d^{4} J_{1} M_{1}\right|_{F_{1,1}}= & -\frac{1}{24 \xi} \operatorname{tr}\left\{-\frac{2}{\left(\mathrm{e}^{\varepsilon m}+1\right)\left(\mathrm{e}^{\varepsilon m}-1\right)}\right. \\
& \left.-\frac{\mathrm{e}^{2 \varepsilon m}}{\left(\mathrm{e}^{\varepsilon m}+1\right)^{3}\left(1-\mathrm{e}^{\varepsilon m}\right)}-\frac{\mathrm{e}^{2 \varepsilon m}}{\left(\mathrm{e}^{\varepsilon m}+1\right)\left(1-\mathrm{e}^{\varepsilon m}\right)^{3}}\right\} .
\end{aligned}
$$


Doing this sum we reconstruct the answer (which can be also obtained independently using diagram technique):

$$
\begin{aligned}
F_{1,1} & =-\frac{1}{12 \xi} \operatorname{tr} \frac{3 \mathrm{e}^{2 \varepsilon m}-1}{\left(\mathrm{e}^{2 \varepsilon m}-1\right)^{3}} \\
& =\left.\operatorname{tr}\left\{-\frac{1}{24 \tilde{\xi}} \frac{\partial^{2}}{\partial m^{2}} \frac{1}{\varepsilon^{2}} \frac{1}{\mathrm{e}^{\varepsilon m}-1}+\frac{1}{6 \tilde{\xi}} \frac{1}{\mathrm{e}^{\varepsilon m}-1}\right\}\right|_{\substack{\text { symmetrized } \\
\mathrm{e}^{\varepsilon m} k \rightarrow-\mathrm{e}^{\varepsilon m m_{k}}}},
\end{aligned}
$$

where we again substitute $\tilde{\xi}=2 \xi=\varepsilon^{-3}$ in order to compare with the Kontsevich model answers. That is, the form (5.54) is explicitly reproduced. The first coefficient, $\frac{1}{24}$, is just the Kontsevich index, and the second term corresponds to the unique reduction of the torus, i.e. the sphere with an additional factor $1 / 3$ ! due to reduction.

This rather simple, but instructive example shows that the hermitian matrix model might be able to offer some new insight in the fine structure of moduli space.

\section{Discussion}

We would like to mention at the end one more approach to the hermitian matrix model which is based on its interpretation as an integrable system. The appropriate hierarchy for the hermitian matrix model is the Toda-chain one [32, 28] and its partition function can be represented as the corresponding $\tau$-function. This $\tau$-functin obeys 333 a set of discrete Virasoro constraints quite similarly to the continuum partition function [34]. The relation between these two sets of the Virasoro constraints was studied in Ref. [25].

As is discussed in Ref. [25], these Virasoro constraints for the hermitian matrix model are nothing but the loop equation (2.12). Therefore, the explicit solutions found in the present paper are simultaneously solutions of the Virasoro constraints. We do not refer, however, to the fact that the partition function (2.1) is the $\tau$-function. In the double scaling limit this relation gives a powerful computational method to obtain higher orders of the genus expansion based on the Gelfand-Dikii technique. Its analogue for the hermitian matrix model away from the double scaling limit is yet missing. We think that our explicit calculations prompt that such a technique should exist.

Acknowledgements We thank A. Mironov for interesting and valuable discussions. L. Ch. would like to thank for hospitality Professor P.K.Mitter and Laboratoire de Physique Théorique et des Hautes Énergies. Yu. M. thanks the theoretical physics department of UAM for the hospitality at Madrid. 


\section{Appendix A The d.s.l. of complex matrix model}

The partition function of the complex matrix model and the definition of its correlators were given in Section 4.4. In the same way as for the hermitian one matrix model, one can obtain the correlators from the free energy by application of the loop insertion operator, $\frac{d}{d V_{C}(p)}$ :

$$
W^{C}\left(p_{1}, \ldots, p_{s}\right)=\frac{d}{d V_{C}\left(p_{s}\right)} \frac{d}{d V_{C}\left(p_{s-1}\right)} \ldots \frac{d F^{C}}{d V_{C}\left(p_{1}\right)}
$$

where

$$
\frac{d}{d V_{C}(p)} \equiv-\sum_{j=1}^{\infty} \frac{j}{p^{2 j+1}} \frac{d}{d g_{j}}
$$

In Ref. [19 an iterative scheme for calculating $W_{g}^{C}(p)$ for any genus $g$ starting from $W_{0}^{C}(p)$ was developed. The idea was very much the same as described in Section 3.2 . The basic ingredient was the loop equation for the complex matrix model:

$$
\oint_{P} \frac{d \omega}{4 \pi i} \frac{V_{C}^{\prime}(\omega) \omega}{p^{2}-\omega^{2}} W^{C}(\omega)=\left[W^{C}(p)\right]^{2}+\frac{1}{N^{2}} W^{C}(p, p),
$$

which after insertion of the genus expansion of the correlators reads

$$
\left\{\hat{K}_{C}-2 W_{0}^{C}(p)\right\} W_{g}^{C}(p)=\sum_{g^{\prime}=1}^{g-1} W_{g^{\prime}}^{C}(p) W_{g-g^{\prime}}^{C}(p)+\frac{d}{d V_{C}(p)} W_{g-1}^{C}(p) \text {. }
$$

The operator $\hat{K}_{C}$ is given by

$$
\hat{K}_{C} f(p) \equiv \oint_{P} \frac{d \omega}{4 \pi i} \frac{V_{C}^{\prime}(\omega) \omega}{p^{2}-\omega^{2}} f(\omega)
$$

where $V_{C}(\omega)=\sum_{j} g_{j} \omega^{2 j} / j$ and $P$ is a path which encloses the singularities of $W_{C}(p)$. It was assumed that these consisted of only one cut $[-\sqrt{z}, \sqrt{z}$ on the real axis which in the eigenvalue picture corresponds to assuming that the eigenvalue density for $\phi^{\dagger} \phi$ has support only on the interval $[0, z]$.

To characterize the matrix model potential, instead of the couplings $g_{j}$, the following moments were introduced

$$
\begin{aligned}
N_{k} & =\oint_{P} \frac{d \omega}{4 \pi i} \frac{V_{C}^{\prime}(\omega)}{w^{2 k+1}\left(w^{2}+c\right)^{1 / 2}} & & k \geq 0, \\
I_{k} & =\oint_{P} \frac{d \omega}{4 \pi i} \frac{\omega V_{C}^{\prime}(\omega)}{\left(w^{2}+c\right)^{k+1 / 2}} & & k \geq 0 .
\end{aligned}
$$

For the complex matrix model the $m^{\text {th }}$ multi-critical point is characterized by the eigenvalue density for $\phi^{\dagger} \phi$ having a zero of order $(m-1)$ at the endpoint $z$ of its support. 
It is easily shown that the condition for being at this point is $I_{1}=I_{2}=\ldots=I_{m-1}=0$ and $I_{m} \neq 0$. In Ref. [19] it was proven by induction that $W_{g}^{C}(p)$ can be written as

$$
W_{g}^{C}(p)=\sum_{n=1}^{3 g-1} \mathcal{A}_{g}^{(n)} X^{(n)}(p)+\sum_{m=1}^{g} \mathcal{D}_{g}^{(m)} Y^{(m)}(p) .
$$

The basis vectors $X^{(n)}(p)$ and $Y^{(n)}(p)$ fulfill

$$
\begin{aligned}
\left\{\hat{K}_{C}-2 W_{0}^{C}(p)\right\} X^{(n)}(p) & =\frac{1}{\left(p^{2}-z\right)^{n}}, \\
\left\{\hat{K}_{C}-2 W_{0}^{C}(p)\right\} Y^{(n)}(p) & =\frac{1}{p^{2 n}}
\end{aligned}
$$

and are given by

$$
\begin{aligned}
& X^{(n)}(p)=\frac{1}{I_{1}}\left\{\Phi^{(n)}(p)-\sum_{k=1}^{n-1} X^{(k)}(p) I_{n-k+1}\right\}, \\
& Y^{(n)}(p)=\frac{1}{N_{0}}\left\{\Omega^{(n)}(p)-\sum_{k=1}^{n-1} Y^{(k)}(p) N_{n-k}\right\}
\end{aligned}
$$

where

$$
\begin{aligned}
\Phi^{(n)}(p) & =\frac{1}{\left(p^{2}-z\right)^{n+1 / 2}}, \\
\Omega^{(n)}(p) & =\frac{1}{p^{2 n}\left(p^{2}-z\right)^{1 / 2}} .
\end{aligned}
$$

The iteration process for determining $W_{g}^{C}(p)$ is quite similar to the hermitian matrix model. To start one needs an expression for $W_{0}^{C}(p, p)$. Using a trick similar to (3.22), one finds

$$
W_{0}^{C}(p, p)=\frac{z^{2}}{16 p^{2}\left(p^{2}-z\right)^{2}} .
$$

In the general step one makes use of the following rewriting of the loop insertion operator

$$
\frac{d}{d V_{C}(p)}=\sum_{i} \frac{d I_{i}}{d V_{C}(p)} \frac{\partial}{\partial I_{i}}+\sum_{j} \frac{d N_{j}}{d V_{C}(p)} \frac{\partial}{\partial N_{j}}+\frac{d z}{d V_{C}(p)} \frac{\partial}{\partial z}
$$

where

$$
\begin{aligned}
\frac{d I_{i}}{d V_{C}(p)}= & -i \Phi^{(i)}(p)-(i+1 / 2)\left\{\Phi^{i+1}(p)-\frac{I_{i+1}}{I_{1}} \Phi^{(1)}(p)\right\} z, \\
\frac{d N_{j}}{d V_{C}(p)}= & \frac{1}{2}\left\{-(2 j+1) \Omega^{(j+1)}(p)-\frac{1}{p^{2 j}\left(p^{2}+c\right)^{3 / 2}}\right\} \\
& -\frac{1}{2}\left\{\frac{1}{c} \sum_{l=0}^{j} N_{j-l} \frac{(-1)^{l}}{c^{l}}+\frac{(-1)^{j+1}}{c^{j+1}} I_{1}\right\} \frac{d z}{d V_{C}(p)}, \\
\frac{d z}{d V_{C}(p)}= & \frac{z}{I_{1}} \phi^{(1)}(p) .
\end{aligned}
$$


Calculating the right hand side of the loop equation one gets an expression involving fractions of the type $p^{-2 n}\left(p^{2}-z\right)^{-m}$. Decomposing these fractions into fractions of the type $p^{-2 i},\left(p^{2}-z\right)^{-j}$ allows one to identify immediately the coefficients $\mathcal{A}_{g}^{(n)}$ and $\mathcal{D}_{g}^{(n)}$.

Let us now modify the iterative procedure so that it gives us directly the result in the double scaling limit. The double scaling limit of the correlators for the $m^{\text {th }}$ multi-critical model is obtained by fixing the ratio of any given coupling and the first one to its critical value and scaling $p^{2}$ and $z$ as in (4.1) and (4.2). It is easy to convince oneself that, just as it was the case for the hermitian one matrix model, adjusting the iterative procedure to give only the double scaling relevant results amounts to starting from the double scaled version of $W_{0}^{C}(p, p)$ and to discarding all operators in $d / d V_{C}(p)$ which do not lower the power of $a$ by $m+3 / 2$. One finds

$$
W_{0}^{C}(p, p)=\frac{z_{c}}{16\left(p^{2}-z\right)^{2}} \quad(\text { d.s.l. })
$$

and the loop insertion operator reduces to

$$
\frac{d}{d V_{C}(p)}=\sum_{i} \frac{d I_{i}}{d V_{C}(p)} \frac{\partial}{\partial I_{i}}+\frac{d z}{d V_{C}(p)} \frac{\partial}{\partial z} \quad \text { (d.s.l.) }
$$

where

$$
\begin{aligned}
& \frac{d I_{i}}{d V_{C}(p)}=-(i+1 / 2)\left\{\Phi^{i+1}(p)-\frac{I_{i+1}}{I_{1}} \Phi^{(1)}(p)\right\} z_{c} \quad(\text { d.s.l }) \\
& \frac{d z}{d V_{C}(p)}=\frac{z_{c}}{I_{1}} \Phi^{(1)}(p) \quad(\text { d.s.l. }) .
\end{aligned}
$$

Let us compare the formulas $(\mathrm{A.20})-(\mathrm{A.23}$ with the corresponding formulas for the double scaling limit of the symmetric hermitian one matrix model (4.40) - (4.43). Noticing that $d_{c}^{2}=4 z_{c}$ and that in the double scaling limit

$$
M_{k}=d_{c}^{k-1} I_{k}
$$

we see that in this limit $W_{0}^{C}(p, p)=1 / 4 \cdot W_{0}^{(S)}(p, p), d / d V_{C}(p)=1 / 4 \cdot d / d V_{S}(p)$ and $X^{(n)}(p)=\tilde{\chi}^{(n)}(p)$. Since the loop equation looks the same for the complex and hermitian matrix models (cf. Eqs. (2.15) and (A.4)), we thus have

$$
W_{g}^{C}\left(p_{1}, \ldots, p_{s}\right)=\frac{1}{4^{g+s-1}} W_{g}^{(S)}\left(p_{1}, \ldots, p_{s}\right) \quad(\text { d.s.l. }) .
$$

Furthermore, since the generating functional is related to the free energy in the same way for the two models, we also have

$$
F_{g}^{C}=\frac{1}{4^{g-1}} F_{g}^{(S)} \quad \text { (d.s.l.) } .
$$


Hence, the hermitian and complex one matrix models are equivalent in the double scaling limit. However, away from the double scaling, we clearly have to distinct models. We draw the attention of the reader to the fact that no assumptions about the type of critical behaviour entered the arguments of this appendix.

\section{References}

[1] E. Brézin, C. Itzykson, G. Parisi and J.B. Zuber, Commun. Math. Phys. 59 (1978) 35.

[2] G. 't Hooft, Nucl. Phys. B72 (1974) 461.

[3] J. Ambjørn, B. Durhuus and J. Frölich, Nucl. Phys. B257[FS14] (1985) 433;

F. David, Nucl. Phys. B257[FS14] (1985) 45;

V.A. Kazakov, Phys. Lett. 150B (1985) 282;

V.A. Kazakov, I.K. Kostov and A.A. Migdal, Phys. Lett. 157B (1985) 295.

[4] V.A. Kazakov, Mod. Phys. Lett. A4 (1989) 2125.

[5] E. Brézin and V.A. Kazakov, Phys. Lett. 236B (1990) 144;

M. Douglas and S. Shenker, Nucl. Phys. B335 (1990) 635;

D. Gross and A.A. Migdal, Phys. Rev. Lett. 64 (1990) 127.

[6] D. Gross and A.A. Migdal, Nucl. Phys. B340 (1990) 333;

T. Banks, M. Douglas, N. Seiberg and S. Shenker, Phys. Lett. 238B (1990) 279;

M. Douglas, Phys. Lett. 238B (1990) 176.

[7] E. Witten Nucl. Phys. B340 (1990) 281, Surveys in Diff. Geom. 1 (1991) 243;

R. Dijkgraaf and E. Witten, Nucl. Phys. B342 (1990) 486.

[8] J. Horne, Mod. Phys. Lett. A5 (1990) 2127.

[9] M.L. Kontsevich, Funk. Anal. \& Prilozh. 25 (1991) 50 (in Russian), Intersection Theory on the Moduli Space of Curves and the Matrix Airy Function, preprints MPI/91-47 and MPI/91-77.

[10] D. Bessis, Commun. Math. Phys. 69 (1979) 147;

D. Bessis, C. Itzykson, G. Parisi and J.B. Zuber, Adv. Appl. Math. 1 (1980) 109.

[11] A.A. Migdal, Phys. Rep. 102 (1983) 199.

[12] S.R. Wadia, Phys. Rev. D24 (1981) 970.

[13] F. David, Mod. Phys. Lett. A5 (1990) 1019.

[14] J. Ambjørn and Yu. Makeenko, Mod. Phys. Lett., A5 (1990) 1753.

[15] J. Ambørn, L. Chekhov and Yu. Makeenko, Phys. Lett. 282B (1992) 341.

[16] D.J. Gross and M.J. Newman, Phys. Lett. 266B (1991) 291.

[17] C. Itzykson and J.-B. Zuber, Int. J. Mod. Phys. A7 (1992) 5661.

[18] L. Chekhov and Yu. Makeenko, Phys. Lett. 278B (1992) 271.

[19] J. Ambjørn, C.F. Kristjansen and Yu. Makeenko Mod. Phys. Lett. A7 (1992) 3187.

[20] L. Chekhov, Matrix Model for Discretized Moduli Space, Steklov preprint SMI-92-04 (May, 1992). 
[21] Yu. Makeenko, Mod. Phys. Lett. (Brief Reviews) A6 (1991) 1901 and references therein.

[22] S. Dalley, C. Johnson and T. Morris, Nucl. Phys. B368 (1992) 625, 655.

[23] J. Ambjørn, J. Jurkiewicz and Yu. Makeenko, Phys. Lett. B251 (1990) 517.

[24] C. Bachas and P.M.S. Petropoulos, Phys. Lett. 247B (1990) 363.

[25] Yu. Makeenko, A. Marshakov, A. Mironov and A. Morozov, Nucl. Phys. B356 (1991) 574.

[26] E. Witten, On the Kontsevich Model and other Models of Two-Dimensional Gravity, preprint IASSNS-HEP-91/24 (June, 1991).

[27] A. Marshakov, A. Mironov and A. Morozov, Phys. Lett. B274 (1992) 280;

D.J. Gross and M.J. Newman, Nucl. Phys. B380 (1992) 168.

[28] S. Kharchev, A. Marshakov, A. Mironov and A. Morozov, Generalized Kontsevich Model versus Toda hierarchy, preprint FIAN/TD-03/92 (February, 1992).

[29] R.C. Penner, Comm. Math. Phys. 113 (1987), 299; J. Diff. Geom. 27 (1988) 35.

[30] L. Chekhov and Yu. Makeenko, Mod. Phys. Lett. A7 (1992) 1223.

[31] Yu. Makeenko and G. Semenoff, Mod. Phys. Lett., A6 (1991) 3455;

Yu. Makeenko, in Proc. XXV Int. Symp. on the Theory of Elementary Part., Gösen, Germany, September 1991, ed. H.J. Kaiser, p. 275.

[32] A. Gerasimov, A. Marshakov, A. Mironov, A. Morozov and A. Orlov, Nucl. Phys. B357 (1991) 565;

E.J. Martinec, Commun. Math. Phys. 138 (1991) 437;

L. Alvarez-Gaumé, C. Gomez and J. Lacki, Phys. Lett. 253B (1991) 56.

[33] J. Ambjørn et al. Ref. [23];

A. Gerasimov et al. Ref. [32];

H. Itoyama and Y. Matsuo, Phys.Lett. B255 (1991) 202;

A. Mironov and A. Morozov, Phys. Lett. 252B (1990) 47.

[34] M. Fukuma, H. Kawai and R. Nakayama, Int. J. Mod. Phys. A6 (1991) 1385;

R. Dijkgraaf, E. Verlinde and H. Verlinde, Nucl. Phys. B348 (1991) 435. 